\title{
A Covariance Shaping Framework for Linear Multiuser Detection
}

\author{
Yonina C. Eldar, Member, IEEE, and Shlomo Shamai (Shitz), Fellow, IEEE
}

\begin{abstract}
A new class of linear multiuser receivers, referred to as the covariance shaping multiuser (CSMU) receiver, is proposed, for suppression of interference in multiuser wireless communication systems. This class of receivers is based on the recently proposed covariance shaping least-squares estimator, and is designed to minimize the total variance of the weighted error between the receiver output and the observed signal, subject to the constraint that the covariance of the noise component in the receiver output is proportional to a given covariance matrix, so that we control the dynamic range and spectral shape of the output noise. Some of the well-known linear multiuser receivers are shown to be special cases of the CSMU receiver. This allows us to interpret these receivers as the receivers that minimize the total error variance in the observations, among all linear receivers with the same output noise covariance, and to analyze their performance in a unified way.

We derive exact and approximate expressions for the probability of bit error, as well as the asymptotic signal-to-interference+noise ratio in the large system limit. We also characterize the spectral efficiency versus energy-per-information bit of the CSMU receiver in the wideband regime.

Finally, we consider a special case of the CSMU receiver, equivalent to a mismatched minimum mean-squared error (MMSE) receiver, in which the channel signal-to-noise ratio (SNR) is not known precisely. Using our general performance analysis results, we characterize the performance of the mismatched MMSE receiver. We then treat the case in which the SNR is known to lie in a given uncertainty range, and develop a robust mismatched MMSE receiver whose performance is very close to that of the MMSE receiver over the entire uncertainty range.
\end{abstract}

Index Terms-Code-division multiple access (CDMA), covariance shaping, mismatched minimum mean-squared error (MMSE), multiuser detection, noise shaping, robust MMSE.

\section{INTRODUCTION}

$\mathbf{I}$ $\mathrm{N}$ recent years, there has been increased interest in wireless communication systems, as is reflected by the extensive literature in the area and the merging standards for practical systems; see [1] and references therein. Intensive efforts have been invested in developing receivers for suppressing interference in these systems.

Direct-sequence spread-spectrum code-division multiple access (CDMA) has many desirable features; dynamic channel

Manuscript received May 20, 2003; revised March 3, 2005. This work was supported in part by the European Union 6th Framework Program, via the NEWCOM Network of Excellence. The material in this paper was presented in part at the IEEE International Symposium on Information Theory, Yokohama, Japan, June/July 2003.

The authors are with the Department of Electrical Engineering, TechnionIsrael Institute of Technology, Technion City, Haifa 32000, Israel (e-mail: yonina@ee.technion.ac.il; sshlomo@ee.technion.ac.il).

Communicated by D. N.C. Tse, Associate Editor for Communications.

Digital Object Identifier 10.1109/TIT.2005.850049 sharing, robustness to channel impairments, graceful degradation, and more. These advantages result from the assignment of signature waveforms with large time-bandwidth product to every potential user of the system. Each user transmits information by modulating a signature sequence. Multiuser receivers for detection of CDMA signals try to mitigate both the background noise and the multiple-access interference (MAI). Since the optimal receiver for detecting the symbols transmitted by each of the users has exponential complexity [2], [3], several suboptimal linear receivers based on single-user decoding have been proposed, which are practically appealing [4], [5]. These include the linear minimum mean-squared error (MMSE) receiver [6]-[9], the decorrelator receiver [10], [11], the matchedfilter (MF) receiver, and the orthogonal multiuser (OMU) receiver [12], [13]. Computationally efficient approximations of the decorrelator and MMSE receivers have been developed in [14], [15].

In this paper, we propose a broad class of linear multiuser receivers which we refer to as the covariance shaping multiuser (CSMU) receiver. As we show in Section IV-B, this class of receivers is very general, and includes the MMSE, decorrelator, MF, OMU, and the Müller-Verdú [15] receivers as special cases. The CSMU receiver is based on the recently proposed covariance shaping least-squares (CSLS) estimator [16], [17], discussed in Section III. The CSLS estimator is a linear estimator for the unknown deterministic parameters in a linear model, and is directed at improving the performance of the traditional least-squares estimator by choosing the estimator to minimize the (weighted) total error variance in the observations, subject to the constraint that the covariance of the estimation error is proportional to some given covariance matrix $\boldsymbol{R}$, so that we control the dynamic range and spectral shape of the covariance of the estimation error. A difficulty often encountered in least-squares estimation is that the error in the estimation can have a large variance and a covariance structure with a very high dynamic range. As shown in [16], the CSLS estimator can often result in lower mean-squared error (MSE) than the least-squares estimator.

To develop the class of CSMU receivers, we view multiuser receivers as demodulators, directed at extracting good estimates of the coded symbols of each user, which are then used as inputs to the individual channel decoders [9]. As we show in Section IV, the multiuser detection problem can be formulated as the problem of estimating an unknown set of parameters observed through a linear transformation and corrupted by additive white noise. The CSMU receiver results from using a CSLS estimator to estimate these parameters. 
Three equivalent representations of the new receiver are developed in Section IV. In the first, the receiver consists of a bank of correlators with correlating vectors with Gram matrix $\boldsymbol{R}$, that are closest in a least-squares sense to the signature vectors. In the second, the receiver consists of a decorrelator receiver followed by a weighted MMSE covariance shaping transformation [16], [17], that is designed to optimally shape the covariance of the decorrelator outputs prior to detection, by minimizing the weighted MSE between the vector output of the decorrelator and the output of the shaping transformation. In the third, the receiver consists of an MF receiver followed by an MMSE shaping transformation, which minimizes the MSE between the vector output of the MF and the output of the shaping transformation. These three representations are mathematically equivalent, but they provide further insight into the CSMU receiver, and may have different implications in terms of implementation.

In Section V, we evaluate the performance of the CSMU receiver. We first derive exact and approximate expressions for the probability of bit error. We then analyze the asymptotic signal-to-interference+noise ratio (SINR) at the output of the CSMU receiver for a broad class of covariance matrices $\boldsymbol{R}$, assuming random Gaussian signatures and equal power users. As we show, the output SINR converges to a deterministic limit, for which an explicit expression is given. The known limiting SINRs of the MMSE, decorrelator, MF, OMU, and Müller-Verdú receivers [4], [5], [18], [19], [1], [20], [12], [15] can be obtained as special cases of our general SINR expression. Based on the asymptotic SINR, we derive the spectral efficiency of the CSMU receiver, which is the number of bits per chip that can be transmitted reliably [19], [21]. In Section $\mathrm{V}-\mathrm{C}$, we consider an application of the asymptotic results to the problem of developing an optimal convex combination of the $\mathrm{MF}$ and decorrelator receivers, that maximizes the asymptotic SINR. Since the dependence of the resulting receiver on the signal-to-noise ratio (SNR) is only through the convex combination, this allows us to develop a low-complexity receiver in the presence of known SNR fluctuations.

As demonstrated in [19], CDMA systems often operate in the wideband regime where the spectral efficiencies are relatively low. In [22], it was shown that the fundamental limits of such systems can be characterized by the minimum signal energy-per-information bit $\frac{E_{b}}{N_{0}}$ min required for reliable communication, and the wideband slope $\mathcal{S}_{0}$ of the spectral efficiency curve, as a function of $\frac{E_{b}}{N_{0}}$, at $\frac{E_{b}}{N_{0}}$ min. In Section V-D, we develop closed-form expressions for $\frac{E_{b}}{N_{0}}$ min and $\mathcal{S}_{0}$ when using the CSMU receiver, in the large system limit.

It is well known that the linear MMSE receiver maximizes the SINR among all linear receivers, and therefore typically performs better than the MF, decorrelator and OMU receivers. However, the implementation of the MMSE receiver requires knowledge of the users' amplitudes and the noise variance, which in many practical scenarios may not be known. Even when the users have equal power, the MMSE receiver requires knowledge of the channel SNR. There are many scenarios in which the SNR is not known, or may be changing over time.

In Section VI, we treat a special case of the CSMU receiver, in which the output covariance $\boldsymbol{R}$ is chosen to have a similar structure as the output covariance resulting from using an MMSE re- ceiver, but does not require knowledge of the channel SNR. As we show, the resulting receiver is equivalent to a mismatched MMSE receiver, where the SNR is replaced by an estimated SNR. Since the mismatched MMSE receiver is a special case of the CSMU receiver, we can use our general results to evaluate the exact probability of a detection error, the asymptotic SINR, and the asymptotic $\frac{E_{b}}{N_{0}}$ min and wideband slope $\mathcal{S}_{0}$ of this receiver. Using these performance measures we show that in many cases the MMSE-based CSMU receiver performs similarly to the MMSE receiver, even though it does not require knowledge of the channel SNR.

In Section VI-E, we discuss the case in which the SNR is known to lie in a given uncertainty range, and develop a robust mismatched MMSE receiver whose performance is close to that of the MMSE receiver that knows the SNR, over the entire uncertainty region. We demonstrate through an example that the resulting receiver can significantly improve the performance over the decorrelator receiver.

Before proceeding to the detailed development, in the next section we formulate our problem and present our main results.

\section{PRoblem Formulation}

We denote vectors in $\mathbb{C}^{M}$ ( $M$ arbitrary) by boldface lower case letters, e.g., $\boldsymbol{a}$, where the $m$ th component of $\boldsymbol{a}$ is denoted by $a_{m}$. Matrices in $\mathbb{C}^{N \times M}$ are denoted by boldface upper case letters, e.g., $\boldsymbol{S}$. The range space and null space of the matrix $\boldsymbol{S}$ are denoted by $\mathcal{R}(\boldsymbol{S})$ and $\mathcal{N}(\boldsymbol{S})$, respectively. $\boldsymbol{I}_{M}$ denotes the $M \times M$ identity matrix, $(\cdot)^{*}$ denotes the Hermitian conjugate of the corresponding matrix, $(\cdot)^{\dagger}$ denotes the Moore-Penrose pseudoinverse [23], and $\widehat{(\cdot)}$ denotes an estimated vector or matrix. The $m k$ th element and the $m$ th column of a matrix are denoted by $[\cdot]_{m k}$ and $[\cdot]_{m}$ respectively. $P_{\mathcal{U}}$ denotes the orthogonal projection operator onto the subspace $\mathcal{U}$, and $\mathcal{U}^{\perp}$ denotes the orthogonal complement of $\mathcal{U}$. The sign of $a$ is denoted by $\operatorname{sgn}(a)$, and is equal to 1 for $a \geq 0$ and -1 for $a<0$. A prime attached to a random variable or vector denotes the variable or vector with the conditional mean given the transmitted data subtracted, e.g., $\boldsymbol{a}^{\prime}=\boldsymbol{a}-E\{\boldsymbol{a} \mid \boldsymbol{b}\}$, where $\boldsymbol{b}$ is the vector of transmitted data.

Consider an $M$-user white Gaussian synchronous CDMA system where each user transmits information by modulating a signature sequence. The discrete-time model for the received signal $\boldsymbol{r}$ is given by

$$
r=S A b+n
$$

where $\boldsymbol{S}=\left[\boldsymbol{s}_{1}\left|\boldsymbol{s}_{2}\right| \ldots \mid \boldsymbol{s}_{M}\right]$ is the $N \times M$ matrix of signatures with $\boldsymbol{s}_{m} \in \mathbb{C}^{N}$ being the signature vector of the $m$ th user, $\boldsymbol{A}=\operatorname{diag}\left(A_{1}, \ldots, A_{M}\right)$ is the matrix of received amplitudes with $A_{m}>0$ being the amplitude of the $m$ th user's signal, $\boldsymbol{b}=\left[b_{1}, b_{2}, \ldots, b_{M}\right]^{*}$ is the data vector with $b_{m}$ being the $m$ th user's transmitted symbol, and $\boldsymbol{n}$ is a noise vector whose elements are independent $\mathcal{C N}\left(0, \sigma^{2}\right)$. We assume that all data vectors are equally likely with covariance $\boldsymbol{I}_{N}$, and that the users are transmitting with equal power so that $\boldsymbol{A}=A \boldsymbol{I}_{M}$ for some $A>0$. For concreteness, we also assume that $b_{m} \in\{1,-1\}$.

Based on the observed signal $\boldsymbol{r}$, we design a receiver to detect the information transmitted by each user. The complexity of the 


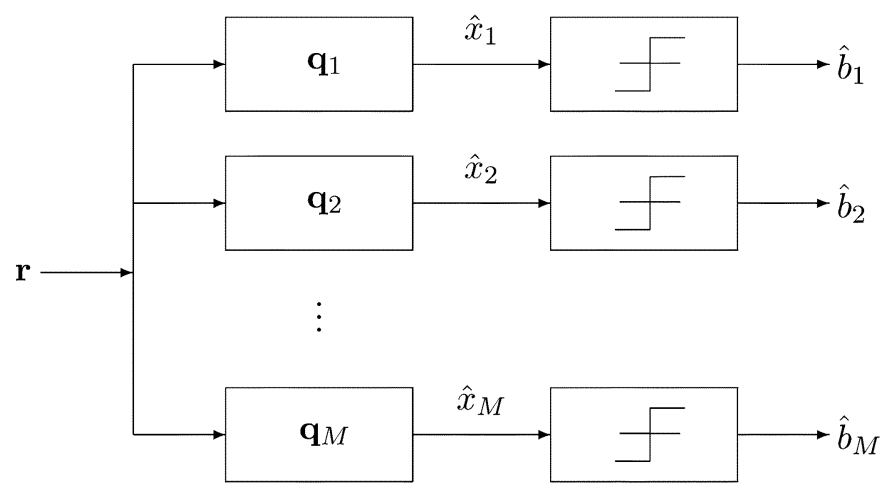

Fig. 1. General linear receiver comprised of a bank of correlators with correlating vectors $\boldsymbol{q}_{m}$ followed by a bank of detectors.

optimal receiver grows exponentially with $M$, and is therefore impractical to implement for large $M$ [2], [3]. We therefore consider suboptimal linear receivers that are easier to implement. Specifically, in this paper we develop receivers that consist of two parts, the signal demodulator which is designed to estimate $\hat{\boldsymbol{x}}=\boldsymbol{A} \boldsymbol{b}$, and a bank of single-user detectors which are comprised of threshold devices. We restrict our attention to linear demodulators so that $\hat{\boldsymbol{x}}$ has the form $\hat{\boldsymbol{x}}=\boldsymbol{Q}^{*} \boldsymbol{r}$ for some matrix $\boldsymbol{Q}$. The $m$ th user's symbol is then detected as $\hat{b}_{m}=\operatorname{sgn}\left(\hat{x}_{m}\right)$ where $\hat{x}_{m}=\boldsymbol{q}_{m}^{*} \boldsymbol{r}$ is the $m$ th component of $\hat{\boldsymbol{x}}$, and $\boldsymbol{q}_{m}=[\boldsymbol{Q}]_{m}$. A receiver of this form can be implemented using a bank of correlators with correlating vectors $\boldsymbol{q}_{m}$, as depicted in Fig. 1.

The observed signal $\boldsymbol{r}$ is related to the unknown vector of parameters $\boldsymbol{x}$ through the linear model

$$
\boldsymbol{r}=\boldsymbol{S} \boldsymbol{x}+\boldsymbol{n} .
$$

Therefore, the design problem associated with Fig. 1 is equivalent to the problem of estimating $\boldsymbol{x}$ in the linear model (2).

Several approaches have emerged to designing linear demodulators, which differ, among other factors, in their assumptions regarding the channel parameters. If the parameters are known, then the demodulator can be designed to maximize the output SINR so that the receiver mitigates optimally both the white noise and the MAI. The optimal receiver of this form is the linear MMSE receiver [6], [7], corresponding to $\boldsymbol{Q}=\boldsymbol{S}\left(\boldsymbol{S}^{*} \boldsymbol{S}+\right.$ $\left.\left(\sigma^{2} / A^{2}\right) \boldsymbol{I}\right)^{-1}$.

If the channel parameters are not known, then the MMSE receiver cannot be implemented. Several linear receivers that do not assume knowledge of the channel parameters have been proposed, and differ in their strategy for estimating $\boldsymbol{x}$ in the model (2).

In the simplest approach, the MAI is ignored and the demodulator is designed to optimally compensate for the white noise on the channel. This method leads to the single-user MF, corresponding to $\boldsymbol{Q}=\boldsymbol{S}$. Although the MF receiver optimally compensates for the white noise, it does not take the structure of the MAI into account.

An alternative strategy is to estimate $\boldsymbol{x}$ using the least-squares estimator [1], [24], which leads to the well-known decorrelator receiver [10], [11], and corresponds to $\boldsymbol{Q}=$ $\boldsymbol{S}\left(\boldsymbol{S}^{*} \boldsymbol{S}\right)^{\dagger}$. The decorrelator optimally rejects the MAI when the signature vectors are linearly independent; however, the inverse operation of the decorrelator may lead to an output noise component with large variance and a covariance structure with a very high dynamic range, resulting in degraded performance.

A third approach is to estimate $\boldsymbol{x}$ by first using a least-squares estimator, and then optimally whitening the noise component in the output of the estimator [13], [12]. This leads to the OMU receiver, and corresponds to choosing $\boldsymbol{Q}=\boldsymbol{S}\left(\left(\boldsymbol{S}^{*} \boldsymbol{S}\right)^{1 / 2}\right)^{\dagger}$. This receiver tries to mitigate both the effect of the white noise and the MAI by optimally whitening the outputs of the decorrelator prior to detection.

Implementing each of the receivers above involves computing an inverse of a matrix, which depends on the signature matrix and possibly the SNR. In cases where the signatures or the SNR vary rapidly with time, computation of the inverse may be expensive. In such settings, it was suggested in [15] to approximate the MMSE receiver (or the decorrelator receiver in the case in which the SNR is not known) by a weighted polynomial matrix, where the weights are chosen to maximize the asymptotic SINR. Assuming equal-power users, the resulting receiver, which we refer to as the Müller-Verdú receiver, corresponds to

$$
\boldsymbol{Q}=\boldsymbol{S} \sum_{\ell=0}^{L} w_{\ell}\left(\boldsymbol{S}^{*} \boldsymbol{S}\right)^{\ell}
$$

where $w_{\ell}$ are the weights.

In this paper, we show that the MMSE, MF, decorrelator, OMU, and Müller-Verdú receivers are actually special cases of the more general class of CSMU receivers, which is based on the CSLS estimator [16], [17]. The CSLS estimator is directed at improving the performance of the traditional least-squares estimator for the unknown parameters $\boldsymbol{x}$ in a linear model, by choosing the estimate of $\boldsymbol{x}$ to minimize the (weighted) total error variance in the observations, subject to the constraint that the covariance of the estimation error is proportional to some given covariance matrix $\boldsymbol{R}$, so that we control the dynamic range and spectral shape of the covariance of the estimation error.

Following [17], [25], we propose using the CSLS estimator to estimate $\boldsymbol{x}$ in (2) which leads to the class of CSMU receivers. This class is quite general since we have the freedom of choosing the output covariance $\boldsymbol{R}$. For specific choices of $\boldsymbol{R}$, the CSMU receiver reduces to some of the well-known multiuser receivers. In particular, we show in Section IV-B that the MMSE, MF, decorrelator, OMU, and Müller-Verdú receivers can all be formulated as CSMU receivers. This provides additional insight and further optimality properties of these receivers. However, the CSMU receiver is more general since we are not constrained to a specific choice of covariance $\boldsymbol{R}$. By choosing different values of $\boldsymbol{R}$ we can generate a variety of different linear multiuser receivers, and analyze their performance in a unified manner. Two specific examples are developed in Sections V-C and VI.

In Section V, we extend the analysis of [12], [20] to the analysis of the asymptotic SINR in the large system limit of the CSMU receiver, for a general class of covariance matrices $\boldsymbol{R}$ and equal power users, which includes the covariance matrices corresponding to the MF, decorrelator, OMU, MMSE, and Müller-Verdú receivers. Specifically, we develop expressions for the asymptotic SINR and asymptotic spectral efficiency in the large system limit for Gaussian signatures and equal power 
users. These results can be used to evaluate the performance of any linear receiver that can be formulated as a CSMU receiver with covariance $\boldsymbol{R}$ that lies in the class we consider. Therefore, for example, this method of analysis can be used as an alternative to methods in [4], [19] for deriving the asymptotic SINR of the MF and MMSE receivers. Our results can also be used to characterize the energy-per-information bit required to achieve a given spectral efficiency, in the wideband regime, for the MF, decorrelator, OMU, and Müller-Verdú receivers.

In Sections V-C and VI, we present two examples of the CSMU receiver. The first example, considered in Section V-C, corresponds to a convex combination of the MF and decorrelator receivers, so that

$$
\boldsymbol{Q}=(1-a) \boldsymbol{S}+a \boldsymbol{S}\left(\boldsymbol{S}^{*} \boldsymbol{S}\right)^{-1}, \quad \text { for some } 0 \leq a \leq 1 .
$$

Since, as we show, this receiver is a special case of the CSMU receiver, its asymptotic performance can be determined from our general asymptotic results of Section V. Specifically, we obtain a simple expression for the asymptotic SINR of the receiver, as a function of the coefficient $a$. We then show that $a$ can be chosen to maximize the asymptotic SINR, resulting in a receiver that performs better than the MF and the decorrelator. This receiver may be useful, for example, if the signatures are known and fixed, however, the SNR varies with time. Since the dependence of the proposed receiver on the SNR is only through the coefficient $a$, updating the receiver in the presence of SNR fluctuations is computationally cheaper then implementing the MMSE receiver, which involves a matrix inversion.

In Section VI, we treat the case in which the SNR is unknown to the receiver, and propose a CSMU receiver for which the output covariance has the same structure as the output covariance of the MMSE receiver. The resulting CSMU receiver can be viewed as an MMSE receiver in which the unknown SNR is replaced by its estimate. Using the analysis results of Section V, we develop exact expressions for the asymptotic performance of this receiver and compare it with that of the MMSE receiver. As we show, if the estimate is close to the true value, then the performance of the proposed receiver can approach the performance of the linear MMSE receiver. We then consider the case in which the SNR is known to lie in a given uncertainty range, and propose a robust receiver whose performance is uniformly close to that of the MMSE receiver, over the entire range.

In the next section, we summarize results from [16], [17] pertaining to the CSLS estimator. We then use these results to develop the class of CSMU receivers in Section IV.

\section{The COVARIANCE SHAPING LEAST-SQUARES ESTIMATOR}

Consider the linear model

$$
y=H x+w
$$

where $\boldsymbol{y}$ is a vector of observations, $\boldsymbol{x}$ is a deterministic vector of unknown parameters, $\boldsymbol{H}$ is a known $n \times m$ matrix, and $\boldsymbol{w}$ is a zero-mean random vector with covariance $C_{w}$.

The CSLS estimate of $\boldsymbol{x}$, denoted $\hat{\boldsymbol{x}}_{\mathrm{CSLS}}$, is chosen to minimize the total variance of the weighted error between $\hat{\boldsymbol{y}}=$
$\boldsymbol{H} \hat{\boldsymbol{x}}_{\mathrm{CSLS}}=\boldsymbol{H} \boldsymbol{G} \boldsymbol{y}$ and $\boldsymbol{y}$, subject to the constraint that the covariance of the error in the estimate $\hat{\boldsymbol{x}}_{\mathrm{CSLS}}$ is proportional to a given covariance matrix $\boldsymbol{R}$. From (3), it follows that the covariance of $\boldsymbol{y}$ is equal to $\boldsymbol{C}_{w}$, so that the covariance of $\hat{\boldsymbol{x}}_{\mathrm{CSLS}}$, which is equal to the covariance of the error in the estimate $\hat{\boldsymbol{x}}_{\mathrm{CSLS}}$, is given by $\boldsymbol{G} \boldsymbol{C}_{w} \boldsymbol{G}^{*}$. Thus, $\hat{\boldsymbol{x}}_{\mathrm{CSLS}}=\boldsymbol{G} \boldsymbol{y}$ is chosen to minimize

$$
\varepsilon_{\mathrm{CSLS}}=E\left\{\left(\boldsymbol{y}^{\prime}-\boldsymbol{H} \boldsymbol{G} \boldsymbol{y}^{\prime}\right)^{*} \boldsymbol{C}_{w}^{-1}\left(\boldsymbol{y}^{\prime}-\boldsymbol{H} \boldsymbol{G} \boldsymbol{y}^{\prime}\right)\right\}
$$

subject to

$$
\boldsymbol{G} \boldsymbol{C}_{w} \boldsymbol{G}^{*}=c^{2} \boldsymbol{R}
$$

where $\boldsymbol{y}^{\prime}=\boldsymbol{y}-E\{\boldsymbol{y}\}, \boldsymbol{R}$ is a given covariance matrix, and $c>0$ is a constant that is either specified in advance, or chosen to minimize the error (4). Since in our detection problem the scaling of $\hat{\boldsymbol{x}}_{\mathrm{CSLS}}$ will not affect the detector output, we restrict our attention to the case in which $c$ is fixed.

The CSLS estimator is summarized in the following theorem.

Theorem 1 (CSLS Estimator [16], [17]): Let $\boldsymbol{x}$ denote the deterministic unknown parameters in the model $\boldsymbol{y}=\boldsymbol{H} \boldsymbol{x}+\boldsymbol{w}$, where $\boldsymbol{H}$ is a known matrix, and $\boldsymbol{w}$ is a zero-mean random vector with covariance $\boldsymbol{C}_{w}$. Let $\hat{\boldsymbol{x}}_{\mathrm{CSLS}}$ denote the CSLS estimator of $\boldsymbol{x}$ that minimizes the error (4) subject to (5), for some $c>0$ and covariance matrix $\boldsymbol{R}$ with $\mathcal{R}(\boldsymbol{R})=\mathcal{N}(\boldsymbol{H})^{\perp}$. Then

$$
\hat{\boldsymbol{x}}_{\mathrm{CSLS}}=c\left(\left(\boldsymbol{R} \boldsymbol{H}^{*} \boldsymbol{C}_{w}^{-1} \boldsymbol{H}\right)^{1 / 2}\right)^{\dagger} \boldsymbol{R} \boldsymbol{H}^{*} \boldsymbol{C}_{w}^{-1} \boldsymbol{y} .
$$

Note that if $\boldsymbol{C}_{w}=\sigma^{2} \boldsymbol{C}$ for some covariance matrix $\boldsymbol{C}$, then choosing $c=\sigma$ results in a CSLS estimator that does not depend on $\sigma^{2}$ which may not be known.

The CSLS estimator can alternatively be expressed as a least-squares estimator followed by a weighted minimum mean-squared error (WMMSE) covariance shaping transformation [17]. Specifically, suppose we estimate the parameters $\boldsymbol{x}$ using the least-squares estimator $\hat{\boldsymbol{x}}_{\mathrm{LS}}=\left(\boldsymbol{H}^{*} \boldsymbol{C}_{w}^{-1} \boldsymbol{H}\right)^{\dagger} \boldsymbol{H}^{*} \boldsymbol{C}_{w} \boldsymbol{y}$. Since $\hat{\boldsymbol{x}}_{\mathrm{LS}}=\boldsymbol{x}+\tilde{\boldsymbol{w}}$, where $\tilde{\boldsymbol{w}}=\left(\boldsymbol{H}^{*} \boldsymbol{C}_{w}^{-1} \boldsymbol{H}\right)^{\dagger} \boldsymbol{H}^{*} \boldsymbol{C}_{w} \boldsymbol{w}$, the covariance of the noise component $\tilde{w}$ in $\hat{x}_{\mathrm{LS}}$ is equal to the covariance of $\hat{\boldsymbol{x}}_{\mathrm{LS}}$, denoted $\boldsymbol{C}_{\hat{\boldsymbol{x}}_{\mathrm{LS}}}$, which is given by $\boldsymbol{C}_{\hat{\boldsymbol{x}}_{\mathrm{LS}}}=\left(\boldsymbol{H}^{*} \boldsymbol{C}_{w}^{-1} \boldsymbol{H}\right)^{\dagger}$. To improve the performance of the least-squares estimator, we consider shaping ${ }^{1}$ the covariance of the noise component in the estimator $\hat{\boldsymbol{x}}_{\mathrm{LS}}$. Thus, we seek a transformation $\boldsymbol{W}$ such that the covariance matrix of $\hat{\boldsymbol{x}}=\boldsymbol{W} \hat{\boldsymbol{x}}_{\mathrm{LS}}$, denoted by $\boldsymbol{C}_{\hat{x}}$, satisfies

$$
\boldsymbol{C}_{\hat{x}}=W \boldsymbol{C}_{\hat{\boldsymbol{x}}_{\mathrm{LS}}} W^{*}=c^{2} \boldsymbol{R}
$$

for some $c>0$. To minimize the distortion to the estimator $\hat{x}_{\mathrm{LS}}$, from all possible transformations $W$ satisfying (6) we choose the one that minimizes the weighted MSE

$$
E\left\{\left(\hat{\boldsymbol{x}}_{\mathrm{LS}}^{\prime}-\boldsymbol{W} \hat{\boldsymbol{x}}_{\mathrm{LS}}^{\prime}\right)^{*} \boldsymbol{D}\left(\hat{\boldsymbol{x}}_{\mathrm{LS}}^{\prime}-\boldsymbol{W} \hat{\boldsymbol{x}}_{\mathrm{LS}}^{\prime}\right)\right\}
$$

${ }^{1}$ When we refer to shaping a random vector $\boldsymbol{a}$, we explicitly mean shaping the noise component in $\boldsymbol{a}$. Equivalently, this corresponds to shaping $\boldsymbol{a}-E\{\boldsymbol{a} \mid \boldsymbol{b}\}$. Similarly, when we say that a random vector $\boldsymbol{a}$ has covariance $\boldsymbol{C}_{a}$ we explicitly mean that the noise component $\boldsymbol{a}-E\{\boldsymbol{a} \mid \boldsymbol{b}\}$ in $\boldsymbol{a}$ has covariance $\boldsymbol{C}_{a}$. 
where $\hat{\boldsymbol{x}}_{\mathrm{LS}}^{\prime}=\hat{\boldsymbol{x}}_{\mathrm{LS}}-E\left\{\hat{\boldsymbol{x}}_{\mathrm{LS}}^{\prime}\right\}$ and $\boldsymbol{D}$ is an arbitrary weighting matrix. The resulting shaping transformation is referred to as a WMMSE shaping transformation. It was shown in [16], [17] that if we choose $\boldsymbol{D}=C_{\hat{x}_{\mathrm{LS}}}^{\dagger}$ in (7), then the resulting estimator $\hat{\boldsymbol{x}}=\boldsymbol{W} \hat{\boldsymbol{x}}_{\mathrm{LS}}$ is equal to $\hat{\boldsymbol{x}}_{\mathrm{CSLS}}$. Thus, the CSLS estimator can be determined by first finding the least-squares estimator $\hat{\boldsymbol{x}}_{\mathrm{LS}}$, and then optimally shaping its covariance using a WMMSE shaping transformation.

The CSLS estimator can also be expressed as an MF estimator followed by MMSE shaping. Consider estimating the parameters $\boldsymbol{x}$ using the transformation $\hat{\boldsymbol{x}}_{\mathrm{MF}}=\boldsymbol{H}^{*} \boldsymbol{C}_{w}^{-1} \boldsymbol{y}$. Then the covariance of the noise component in $\hat{\boldsymbol{x}}_{\mathrm{MF}}$, which is equal to the covariance of $\hat{\boldsymbol{x}}_{\mathrm{MF}}$, is $\boldsymbol{C}_{\hat{\boldsymbol{x}}_{\mathrm{MF}}}=\boldsymbol{H}^{*} \boldsymbol{C}_{w}^{-1} \boldsymbol{H}$. To improve the performance of $\hat{\boldsymbol{x}}_{\mathrm{MF}}$ we choose to shape its covariance, so that we seek a transformation $\boldsymbol{T}$ such that the covariance matrix of $\hat{\boldsymbol{x}}=\boldsymbol{T} \hat{\boldsymbol{x}}_{\mathrm{MF}}$, denoted by $\boldsymbol{C}_{\hat{\boldsymbol{x}}}$, satisfies

$$
\boldsymbol{C}_{\hat{x}}=\boldsymbol{T} \boldsymbol{C}_{\hat{\boldsymbol{x}}_{\mathrm{MF}}} \boldsymbol{T}^{*}=c^{2} \boldsymbol{R}
$$

for some $c>0$. To minimize the distortion to the estimator $\hat{\boldsymbol{x}}_{\mathrm{MF}}$, among all possible transformations $\boldsymbol{T}$ satisfying (8) we choose the one that minimizes the MSE

$$
E\left\{\left(\hat{\boldsymbol{x}}_{\mathrm{MF}}^{\prime}-\boldsymbol{T} \hat{\boldsymbol{x}}_{\mathrm{MF}}^{\prime}\right)^{*}\left(\hat{\boldsymbol{x}}_{\mathrm{MF}}^{\prime}-\boldsymbol{T} \hat{\boldsymbol{x}}_{\mathrm{MF}}^{\prime}\right)\right\}
$$

where $\hat{\boldsymbol{x}}_{\mathrm{MF}}^{\prime}=\hat{\boldsymbol{x}}_{\mathrm{MF}}-E\left\{\hat{\boldsymbol{x}}_{\mathrm{MF}}\right\}$, which we refer to as the MMSE shaping transformation [26]. The resulting estimator $\hat{\boldsymbol{x}}=\boldsymbol{T} \hat{\boldsymbol{x}}_{\mathrm{MF}}$ is equal to $\hat{\boldsymbol{x}}_{\mathrm{CSLS}}$. Thus, the CSLS estimator can also be determined by first finding the MF estimator $\hat{\boldsymbol{x}}_{\mathrm{MF}}$, and then optimally shaping its covariance using an MMSE shaping transformation.

\section{The Covariance Shaping Multiuser Receiver}

We now propose a class of multiuser receivers, referred to as the CSMU receiver, that results from estimating $\boldsymbol{x}$ in the model (2) using a CSLS estimator with $R$ chosen such that $\mathcal{R}(\boldsymbol{R})=$ $\mathcal{N}(\boldsymbol{S})^{\perp}$. This class of receivers provides a unified framework for developing a variety of different linear multiuser receivers, and analyzing their performance.

With $\mathcal{R}(\boldsymbol{R})=\mathcal{N}(\boldsymbol{S})^{\perp}$, the CSLS estimator of $\boldsymbol{x}$ follows from Theorem 1 as

$$
\hat{\boldsymbol{x}}_{\mathrm{CSLS}}=\frac{c}{\sigma}\left(\left(\boldsymbol{R} \boldsymbol{S}^{*} \boldsymbol{S}\right)^{1 / 2}\right)^{\dagger} \boldsymbol{R} \boldsymbol{S}^{*} \boldsymbol{y}=\frac{c}{\sigma} \boldsymbol{C}^{*} \boldsymbol{r}
$$

where

$$
\boldsymbol{C}=\boldsymbol{S R}\left(\left(\boldsymbol{S}^{*} \boldsymbol{S R}\right)^{1 / 2}\right)^{\dagger}
$$

for some covariance matrix $\boldsymbol{R}$. Note, that the scaling of $\hat{\boldsymbol{x}}_{\mathrm{CSLS}}$ will not effect the detector output and therefore can be chosen arbitrarily. In our derivation, we assume that $c=\sigma$ so that the estimator $\hat{\boldsymbol{x}}_{\mathrm{CSLS}}$ of (10) does not depend on $\sigma$, which may be unknown. Henceforth we denote $\hat{\boldsymbol{x}}=\hat{\boldsymbol{x}}_{\mathrm{CSLS}}=\boldsymbol{C}^{*} \boldsymbol{r}$.

We conclude that the CSMU receiver can be implemented using the bank of correlators of Fig. 1 with $\boldsymbol{q}_{m}=\boldsymbol{c}_{m}$ where $\boldsymbol{c}_{m}=[\boldsymbol{C}]_{m}$, and $\boldsymbol{C}$ is given by (11). We refer to the vectors $\boldsymbol{c}_{m}$ as the CSMU vectors.

From the properties of the CSLS estimator, the CSMU demodulator minimizes the total error variance in the received signal subject to the constraint that the covariance of the noise component in the output of the demodulator of Fig. 1 is proportional to a given covariance matrix $\boldsymbol{R}$, so that we control the dynamic range and spectral shape of the noise at the output of the demodulator. The particular shaping $\boldsymbol{R}$ can be tailored to the specific set of signatures.

\section{A. Equivalent Representations of the CSMU Receiver}

We now develop several representations of the CSMU receiver, based on the properties of the CSLS estimator and the CSMU vectors.

1) Correlation Demodulator Representation: We first show that the CSMU receiver can be represented as a correlation demodulator of the form of Fig. 1 with correlating vectors with Gram matrix $\boldsymbol{R}$ that are closest to the signature vectors $\boldsymbol{s}_{m}$, in a least-squares sense.

To this end, we note that the Gram matrix of inner products of the CSMU vectors $\boldsymbol{c}_{m}$ is equal to $\boldsymbol{R}$. Indeed

$$
\begin{aligned}
\boldsymbol{C}^{*} \boldsymbol{C} & =\left(\left(\boldsymbol{R} \boldsymbol{S}^{*} \boldsymbol{S}\right)^{1 / 2}\right)^{\dagger} \boldsymbol{R} \boldsymbol{S}^{*} \boldsymbol{S} \boldsymbol{R}\left(\left(\boldsymbol{S}^{*} \boldsymbol{S} \boldsymbol{R}\right)^{1 / 2}\right)^{\dagger} \\
& =\left(\left(\boldsymbol{R} \boldsymbol{S}^{*} \boldsymbol{S}\right)^{1 / 2}\right)^{\dagger} \boldsymbol{R}\left(\boldsymbol{S}^{*} \boldsymbol{S} \boldsymbol{R}\right)^{1 / 2} \\
& =\left(\left(\boldsymbol{R} \boldsymbol{S}^{*} \boldsymbol{S}\right)^{1 / 2}\right)^{\dagger}\left(\boldsymbol{R} \boldsymbol{S}^{*} \boldsymbol{S}\right)^{1 / 2} \boldsymbol{R} \\
& =\boldsymbol{R},
\end{aligned}
$$

where we used the fact that for any two matrices $\boldsymbol{X}$ and $\boldsymbol{T}$ such that $\mathcal{R}(\boldsymbol{T}) \subseteq \mathcal{N}(\boldsymbol{X})^{\perp}$, we have that $\boldsymbol{X}(\boldsymbol{T} \boldsymbol{X})^{1 / 2}=(\boldsymbol{X} \boldsymbol{T})^{1 / 2} \boldsymbol{X}$ [17].

Among all vectors $\boldsymbol{x}_{m}$ with Gram matrix $\boldsymbol{R}$, the CSMU vectors have the property that they are the closest in a least-squares sense to the vectors $\boldsymbol{s}_{m}$. This follows from [17, Ch. 8] where it is shown that given a set of vectors $\boldsymbol{x}_{m}$ and a Gram matrix $\boldsymbol{R}$ such that $\mathcal{N}(\boldsymbol{R})=\mathcal{N}(\boldsymbol{X})$, where $\boldsymbol{X}$ is the matrix of columns $\boldsymbol{x}_{m}$, the vectors $\boldsymbol{h}_{m}$ with Gram matrix $\boldsymbol{R}$ that minimize the error

$$
\varepsilon_{\mathrm{LS}}=\sum_{m=1}^{M}\left(\boldsymbol{x}_{m}-\boldsymbol{h}_{m}\right)^{*}\left(\boldsymbol{x}_{m}-\boldsymbol{h}_{m}\right)
$$

are given by

$$
\hat{\boldsymbol{h}}_{m}=\left[\boldsymbol{X} \boldsymbol{R}\left(\left(\boldsymbol{X}^{*} \boldsymbol{X} \boldsymbol{R}\right)^{1 / 2}\right)^{\dagger}\right]_{m} .
$$

2) Decorrelator Followed by MMSE Shaping: Since the output of the decorrelator demodulator is equal to $\hat{x}_{\mathrm{LS}}$, from the discussion in Section III it follows that the CSMU receiver can equivalently be implemented as a decorrelator receiver followed by a WMMSE covariance shaping transformation $\hat{T}^{w}$ with weighting $\boldsymbol{C}_{a}^{\dagger}$, as depicted in Fig. 2. Here $\boldsymbol{C}_{a}$ is the covariance of the noise component in the output $\boldsymbol{a}$ of the decorrelator, and the shaping transformation $\hat{\boldsymbol{T}}^{w}$ is designed to optimally shape this covariance prior to detection, and is given from [16] by $\hat{\boldsymbol{T}}^{w}=\left(\boldsymbol{R} \boldsymbol{S}^{*} \boldsymbol{S}\right)^{1 / 2}$.

3) MF Followed by MMSE Shaping: From the representation of the CSLS estimator of Section III, it follows that the CSMU receiver can also be implemented as an MF demodulator followed by an MMSE covariance shaping transformation $\hat{T}$, as depicted in Fig. 3. The transformation $\hat{\boldsymbol{T}}$ is designed to optimally shape the covariance $\boldsymbol{C}_{\tilde{a}}$ of the noise component in the MF output $\tilde{\boldsymbol{a}}=\boldsymbol{S}^{*} \boldsymbol{y}$ prior to detection, and is given by $\left(\left(R S^{*} S\right)^{1 / 2}\right)^{\dagger} \boldsymbol{R}[26]$. 


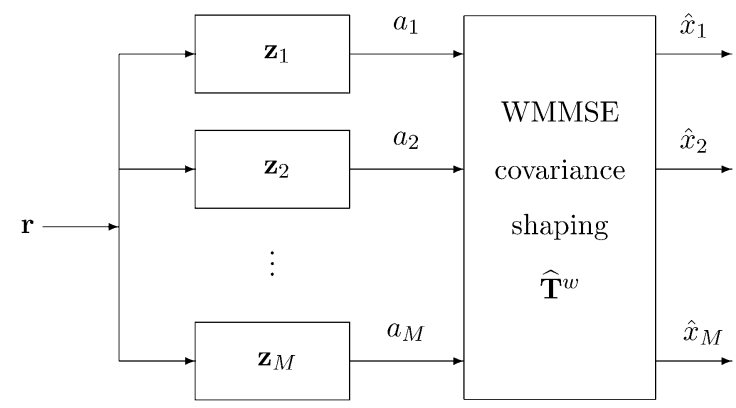

Fig. 2. Representation of the CSMU demodulator in terms of a decorrelator demodulator followed by WMMSE covariance shaping, where $\boldsymbol{z}_{m}=$ $\left[\boldsymbol{S}\left(\boldsymbol{S}^{*} \boldsymbol{S}\right)^{\dagger}\right]_{m}$.

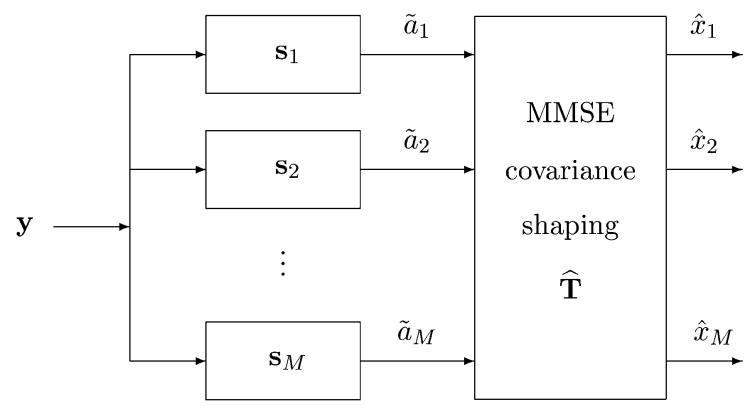

Fig. 3. Alternative representation of the CSMU receiver in terms of an MF demodulator followed by MMSE covariance shaping.

\section{B. Connection With Other Multiuser Receivers}

As we now show, many previously proposed multiuser receivers can be formulated as CSMU receivers with an appropriate choice of $R$.

Let $\hat{\boldsymbol{x}}=\boldsymbol{Q}^{*} \boldsymbol{r}$ be the output of an arbitrary linear receiver. To determine whether the receiver can be formulated as a CSMU receiver, we first compute the output covariance of the receiver, which is given by $\boldsymbol{R}=\sigma^{2} \boldsymbol{Q}^{*} \boldsymbol{Q}$. We then compute the CSMU receiver $\hat{\boldsymbol{x}}_{\mathrm{CSLS}}=\boldsymbol{C}^{*} \boldsymbol{r}$ with covariance $\left(1 / \sigma^{2}\right) \boldsymbol{R}$. If $\hat{\boldsymbol{x}}_{\mathrm{CSLS}}$ is equal to $\hat{\boldsymbol{x}}$, then the original linear receiver can be formulated as a CSMU receiver with output covariance $\left(1 / \sigma^{2}\right) \boldsymbol{R}$. In particular, we have the following proposition.

Proposition 1: Let $\left\{\boldsymbol{s}_{m}, 1 \leq m \leq M\right\}$ denote $M$ signature vectors, and let $\left\{\boldsymbol{q}_{m}, 1 \leq m \leq M\right\}$ denote the correlating vectors of a linear multiuser receiver. Let $\boldsymbol{S}$ and $Q$ denote the matrices of columns $\boldsymbol{s}_{m}$ and $\boldsymbol{q}_{m}$, respectively. If $\boldsymbol{Q}=\boldsymbol{S} \boldsymbol{X}$ for some matrix $\boldsymbol{X}$ satisfying the conditions

$$
\begin{aligned}
\boldsymbol{X} & =\boldsymbol{X}^{*} \\
\mathcal{N}(\boldsymbol{S} \boldsymbol{X}) & =\mathcal{N}(\boldsymbol{S})
\end{aligned}
$$

then the receiver is a CSMU receiver with

$$
R=Q^{*} Q=X S^{*} S X .
$$

Proof: The CSMU vectors corresponding to $\boldsymbol{R}$ of (15) are given from (11) by

$$
\begin{aligned}
\boldsymbol{C} & =\boldsymbol{S} \boldsymbol{X} \boldsymbol{S}^{*} \boldsymbol{S} \boldsymbol{X}\left(\left(\boldsymbol{S}^{*} \boldsymbol{S} \boldsymbol{X} \boldsymbol{S}^{*} \boldsymbol{S} \boldsymbol{X}\right)^{1 / 2}\right)^{\dagger} \\
& =\boldsymbol{S} \boldsymbol{X} \boldsymbol{S}^{*} \boldsymbol{S} \boldsymbol{X}\left(\boldsymbol{S}^{*} \boldsymbol{S} \boldsymbol{X}\right)^{\dagger}=\boldsymbol{S} \boldsymbol{X} P_{\mathcal{R}\left(\boldsymbol{S}^{*} \boldsymbol{S} \boldsymbol{X}\right)} .
\end{aligned}
$$

To prove the proposition we therefore need to show that

$$
\boldsymbol{S} \boldsymbol{X} P_{\mathcal{R}\left(\boldsymbol{S}^{*} \boldsymbol{S} \boldsymbol{X}\right)}=\boldsymbol{S} \boldsymbol{X} .
$$

We first note that $\mathcal{R}\left(\boldsymbol{S}^{*} \boldsymbol{S} \boldsymbol{X}\right)=\mathcal{N}\left(\boldsymbol{X} \boldsymbol{S}^{*} \boldsymbol{S}\right)^{\perp}$. We also note that $\mathcal{N}\left(\boldsymbol{X} \boldsymbol{S}^{*}\right)=\mathcal{N}\left(\boldsymbol{S}^{*}\right)$. This follows from the fact that $\mathcal{R}\left(\boldsymbol{S}^{*}\right)=\mathcal{N}(\boldsymbol{S})^{\perp}$, and since by assumption, $\mathcal{N}(\boldsymbol{S} \boldsymbol{X})=\mathcal{N}(\boldsymbol{S})$, we have that $\mathcal{N}(\boldsymbol{X}) \subseteq \mathcal{N}(\boldsymbol{S})$. Combining these two observations, $\mathcal{R}\left(\boldsymbol{S}^{*} \boldsymbol{S} \boldsymbol{X}\right)=\overline{\mathcal{N}}(\boldsymbol{S})^{\perp}$. Finally, since $\mathcal{N}(\boldsymbol{S} \boldsymbol{X})=\mathcal{N}(\boldsymbol{S})$, we have that $\boldsymbol{S} \boldsymbol{X} P_{\mathcal{N}(\boldsymbol{S})^{\perp}}=\boldsymbol{S X}$, establishing (17).

Using Proposition 1, we can show that the MMSE, decorrelator, MF, OMU, and Müller-Verdú receivers can be formulated as CSMU receivers. Indeed, each of these receivers can be written in the form $\boldsymbol{Q}=\boldsymbol{S} \boldsymbol{X}$ for some $\boldsymbol{X}$ satisfying the conditions of the proposition. Specifically, $\boldsymbol{X}=\left(\boldsymbol{S}^{*} \boldsymbol{S}+\left(\sigma^{2} / A^{2}\right) \boldsymbol{I}\right)^{-1}$ for the MMSE receiver, $\boldsymbol{X}=\left(\boldsymbol{S}^{*} \boldsymbol{S}\right)^{\dagger}$ for the decorrelator receiver, $\boldsymbol{X}=\boldsymbol{I}$ for the MF receiver, $\boldsymbol{X}=\left(\left(\boldsymbol{S}^{*} \boldsymbol{S}\right)^{1 / 2}\right)^{\dagger}$ for the OMU receiver, and $\boldsymbol{X}=\sum_{\ell=0}^{L} w_{\ell}\left(\boldsymbol{S}^{*} \boldsymbol{S}\right)^{\ell}$ for the Müller-Verdú receiver.

We summarize our results regarding the CSMU demodulator in the following theorem.

Theorem 2 (CSMU Demodulator): Let $\left\{\boldsymbol{s}_{m}, 1 \leq m \leq M\right\}$ denote $M$ signature vectors, and let $\left\{\boldsymbol{c}_{m}, 1 \leq m \leq M\right\}$ denote the correlating vectors of the CSMU demodulator. Let $\boldsymbol{S}$ and $\boldsymbol{C}$ denote the matrices of columns $\boldsymbol{s}_{m}$ and $\boldsymbol{c}_{m}$, respectively. Then

$$
\boldsymbol{C}=\boldsymbol{S R}\left(\left(\boldsymbol{S}^{*} \boldsymbol{S R}\right)^{1 / 2}\right)^{\dagger}
$$

where $R$ is any nonnegative definite Hermitian matrix with $\mathcal{R}(\boldsymbol{R})=\mathcal{N}(\boldsymbol{S})^{\perp}$. In addition,

1. any linear receiver with correlating vectors that are the columns of $\boldsymbol{Q}$ where $\boldsymbol{Q}=\boldsymbol{S} \boldsymbol{X}$ for some matrix $\boldsymbol{X}$ with $\boldsymbol{X}=\boldsymbol{X}^{*}$ and $\mathcal{N}(\boldsymbol{S} \boldsymbol{X})=\mathcal{N}(\boldsymbol{S})$ can be formulated as a CSMU receiver with $\boldsymbol{R}=\boldsymbol{X} \boldsymbol{S}^{*} \boldsymbol{S} \boldsymbol{X}$;

2. the vectors $\boldsymbol{c}_{m}$ are the closest vectors with Gram matrix $\boldsymbol{R}$ to the signature vectors $\boldsymbol{s}_{m}$, in the least-squares sense;

3. the CSMU demodulator can be realized by a decorrelator demodulator followed by a WMMSE covariance shaping transformation $\hat{\boldsymbol{T}}^{w}=\left(\boldsymbol{R} \boldsymbol{S}^{*} \boldsymbol{S}\right)^{1 / 2}$;

4. the CSMU demodulator can be realized by an MF demodulator followed by an MMSE covariance shaping transformation $\hat{\boldsymbol{T}}=\left(\left(\boldsymbol{R} \boldsymbol{S}^{*} \boldsymbol{S}\right)^{1 / 2}\right)^{\dagger} \boldsymbol{R}$.

\section{Performance Analysis of the CSMU Receiver}

In this section, we discuss the theoretical performance of the CSMU receiver. We first derive exact and approximate expressions for the probability of detection error for any choice of shaping $\boldsymbol{R}$. We then derive the asymptotic SINR and the spectral efficiency (total capacity per chip) [19], [21] at the output of the CSMU receiver for a certain class of covariance matrices $\boldsymbol{R}$, in the large system limit. Using these results, we develop closed-from expressions for the minimal energy-per-information bit required for reliable transmission and the wideband slope [22], using the CSMU receiver.

\section{A. Exact Probability of Detection Error}

The detector input of the CSMU receiver is

$$
\begin{aligned}
\hat{\boldsymbol{x}} & =\left(\left(\boldsymbol{R} \boldsymbol{S}^{*} \boldsymbol{S}\right)^{1 / 2}\right)^{\dagger} \boldsymbol{R} \boldsymbol{S}^{*} \boldsymbol{r} \\
& =A\left(\boldsymbol{R} \boldsymbol{S}^{*} \boldsymbol{S}\right)^{1 / 2} \boldsymbol{b}+\left(\left(\boldsymbol{R} \boldsymbol{S}^{*} \boldsymbol{S}\right)^{1 / 2}\right)^{\dagger} \boldsymbol{R} \boldsymbol{S}^{*} \boldsymbol{n}
\end{aligned}
$$


Each component of the detector input vector can be decomposed into

$$
\hat{x}_{m}=\hat{x}_{m}^{S}+\hat{x}_{m}^{I}+\hat{x}_{m}^{N}
$$

where the terms

$$
\begin{aligned}
& \hat{x}_{m}^{S}=A\left[\left(\boldsymbol{R} \boldsymbol{S}^{*} \boldsymbol{S}\right)^{1 / 2}\right]_{m m} b_{m} \\
& \hat{x}_{m}^{I}=A \sum_{k \neq m}\left[\left(\boldsymbol{R} \boldsymbol{S}^{*} \boldsymbol{S}\right)^{1 / 2}\right]_{m k} b_{k} \\
& \hat{x}_{m}^{N}=\left[\left(\left(\boldsymbol{R} \boldsymbol{S}^{*} \boldsymbol{S}\right)^{1 / 2}\right)^{\dagger}\right]_{m}^{*} \boldsymbol{R} \boldsymbol{S}^{*} \boldsymbol{n}
\end{aligned}
$$

represent the desired signal, the MAI, and the noise, respectively. Conditioned on $\boldsymbol{b}$, the decision statistic $\hat{x}_{m}$ is Gaussian with mean $\hat{x}_{m}^{S}+\hat{x}_{m}^{I}$ and variance $\sigma^{2}[\boldsymbol{R}]_{m m}$. Taking into consideration all possibilities of $\boldsymbol{b}$, the resulting probability of detection error for the $m$ th user is

$$
\begin{gathered}
P_{m}(\sigma)=\frac{1}{2^{m-1}} \sum_{e_{1} \in\{-1,1\}} \cdots \sum_{\substack{k \in\{-1,1\} \\
k \neq m}} \cdots \sum_{e_{m} \in\{-1,1\}} \\
\mathcal{\mathcal { Q }}\left(\frac{\left[\left(\boldsymbol{R} \boldsymbol{S}^{*} \boldsymbol{S}\right)^{1 / 2}\right]_{m m} A}{\sigma \sqrt{[\boldsymbol{R}]_{m m}}}+\sum_{k \neq m} \frac{\left[\left(\boldsymbol{R} \boldsymbol{S}^{*} \boldsymbol{S}\right)^{1 / 2}\right]_{m k} A}{\sigma \sqrt{[\boldsymbol{R}]_{m m}}} e_{k}\right)
\end{gathered}
$$

where

$$
\mathcal{Q}(v)=\frac{1}{\sqrt{2 \pi}} \int_{v}^{\infty} e^{-t^{2} / 2} d t .
$$

From (21), we see that the probability of detection error of the CSMU detector for the $m$ th user goes to zero as $\sigma \rightarrow 0$ if and only if the argument of each of the $\mathcal{Q}$-functions is positive.

For example, in the special case in which $R=P_{\mathcal{V}}$ where $\mathcal{V}=\mathcal{N}(\boldsymbol{S})^{\perp}$ and all cross correlations of the signature vectors are identically equal to $\rho$, it can be shown that

$$
\begin{aligned}
{\left[\left(\boldsymbol{R} \boldsymbol{S}^{*} \boldsymbol{S}\right)^{1 / 2}\right]_{m k} } & =\left[\left(\boldsymbol{S}^{*} \boldsymbol{S}\right)^{1 / 2}\right]_{m k} \\
& = \begin{cases}\alpha_{1}+(M-1) \alpha_{2}, & \text { if } m=k \\
\alpha_{1}-\alpha_{2}, & \text { if } m \neq k\end{cases}
\end{aligned}
$$

where $\alpha_{1}=(1 / M) \sqrt{1+(M-1) \rho}$ and $\alpha_{2}=(1 / M) \sqrt{1-\rho}$. In this case, the probability of detection error of the CSMU detector for the $m$ th user goes to zero as $\sigma \rightarrow 0$ when

$$
\rho<\frac{3 M-4}{M(M-1)}
$$

\section{B. SINR and Asymptotic Large System Performance}

From (20), the terms $\hat{x}_{m}^{S}, \hat{x}_{m}^{I}$ and $\hat{x}_{m}^{N}$ are mutually independent and zero-mean, and have variances

$$
\begin{aligned}
\operatorname{var}\left(\hat{x}_{m}^{S}\right)= & A^{2}\left[\left(\boldsymbol{R} \boldsymbol{S}^{*} \boldsymbol{S}\right)^{1 / 2}\right]_{m m}^{2} \\
\operatorname{var}\left(\hat{x}_{m}^{I}\right)= & A^{2}\left[\left(\boldsymbol{R} \boldsymbol{S}^{*} \boldsymbol{S}\right)^{1 / 2}\right]_{m}^{*}\left[\left(\boldsymbol{R} \boldsymbol{S}^{*} \boldsymbol{S}\right)^{1 / 2}\right]_{m} \\
& -A^{2}\left[\left(\boldsymbol{R} \boldsymbol{S}^{*} \boldsymbol{S}\right)^{1 / 2}\right]_{m m}^{2} \\
\operatorname{var}\left(\hat{x}_{m}^{N}\right)= & \sigma^{2}[\boldsymbol{R}]_{m m} .
\end{aligned}
$$

The SINR at the detector for the $m$ th user is therefore

$$
\gamma_{m}=\frac{\left[\left(\boldsymbol{R} \boldsymbol{S}^{*} \boldsymbol{S}\right)^{1 / 2}\right]_{m m}^{2}}{\zeta[\boldsymbol{R}]_{m m}+\left[\boldsymbol{R} \boldsymbol{S}^{*} \boldsymbol{S}\right]_{m m}-\left[\left(\boldsymbol{R} \boldsymbol{S}^{*} \boldsymbol{S}\right)^{1 / 2}\right]_{m m}^{2}}
$$

where

$$
\frac{1}{\zeta}=\frac{A^{2}}{\sigma^{2}}
$$

is the received SNR. An alternate form for (26), which will be more convenient for the analysis that follows, is

$$
\gamma_{m}=\frac{1}{1-\frac{\left[\left(\boldsymbol{R} \boldsymbol{S}^{*} \boldsymbol{S}\right)^{1 / 2}\right]_{m m}^{2}}{\boldsymbol{\zeta}[\boldsymbol{R}]_{m m}+\left[\boldsymbol{R S} \boldsymbol{S}^{*}\right]_{m m}}}-1
$$

Assuming $x_{m}^{I}+x_{m}^{N}$ is Gaussian, the probability of error can then be approximated as

$$
P_{m}(\sigma) \approx \mathcal{Q}\left(\sqrt{\gamma_{m}}\right)
$$

where $\mathcal{Q}(\cdot)$ is defined in (22). At low SNR, the Gaussian approximation is acceptable because Gaussian noise is the dominant impairment. However, at high SNR, the discrete distribution of the MAI is poorly approximated by a Gaussian distribution, especially at the tails of the distribution where the bit-error rate (BER) is determined. Thus, we do not expect (29) to be particularly accurate at high SNR. In Section VI, we compare the accuracy of the Gaussian approximation and that of the exact BER expression, in the context of a concrete example of the CSMU receiver.

In the remainder of this section, we focus on the large-system performance of the CSMU receiver for a class of output covariance matrices $R$, assuming equal power users.

Theorem 3 below characterizes the SINR and the spectral efficiency of the CSMU receiver in the large system limit with $\beta \triangleq M / N$ held constant, when random Gaussian signatures and accurate power control are used. For analytical tractability, we focus on output covariance matrices $\boldsymbol{R}$ that have the same eigenvector matrix as $\boldsymbol{S}^{*} \boldsymbol{S}$. Thus, if $\boldsymbol{S}^{*} \boldsymbol{S}$ has an eigendecomposition $\boldsymbol{S}^{*} \boldsymbol{S}=\boldsymbol{V} \Lambda \boldsymbol{V}^{*}$, where $\boldsymbol{V}$ is an $M \times M$ unitary matrix and $\Lambda$ is an $M \times M$ diagonal matrix with diagonal elements $\lambda_{m}$, then $\boldsymbol{R}=\boldsymbol{V} \Sigma \boldsymbol{V}^{*}$, where $\Sigma$ is an $M \times M$ diagonal matrix with diagonal elements $d_{m}$. We further assume that the eigenvalues $d_{m}$ of $\boldsymbol{R}$ can be represented as $d_{m}=t\left(\lambda_{m}\right)$ where $t(\cdot)$ is a continuous function on an interval $\mathcal{I}=[a, b]$ where $a<\eta_{1}$ and $b>\eta_{2}$, with

$$
\begin{aligned}
& \eta_{1}=(1-\sqrt{\beta})^{2} \\
& \eta_{2}=(1+\sqrt{\beta})^{2} .
\end{aligned}
$$

Theorem 3: Let the elements of the $N \times M$ signature matrix $S$ be independent $\mathcal{C N}(0,1 / N)$, and let the matrix of amplitudes $\boldsymbol{A}=A \boldsymbol{I}$. Let $\boldsymbol{S}^{*} \boldsymbol{S}=\boldsymbol{V} \Lambda \boldsymbol{V}^{*}$ where $\Lambda$ is a diagonal matrix with diagonal elements $\lambda_{m}$, let $\boldsymbol{R}=\boldsymbol{V} \Sigma \boldsymbol{V}^{*}$ be a covariance matrix with eigenvalues $d_{m}=t\left(\lambda_{m}\right)$ where $t(\cdot)$ is a continuous function on an interval $\mathcal{I}=[a, b]$ with $a<\eta_{1}$ and $b>\eta_{2}$, where $\eta_{1}$ and $\eta_{2}$ are defined by (30), and $\mathcal{R}(\boldsymbol{R})=\mathcal{N}(\boldsymbol{S})^{\perp}$. Then in the limit, as $M \rightarrow \infty$ with $\beta \triangleq M / N$ held constant we have the following.

1. The SINR for each user at the CSMU receiver output converges almost surely to $\gamma=\alpha /(1-\alpha)$ where

$$
\alpha=\frac{E^{2}\left\{[t(\lambda) \lambda]^{1 / 2}\right\}}{E\{t(\lambda)(\lambda+\zeta)\}}
$$


$1 / \zeta=A^{2} / \sigma^{2}$ is the received SNR, and the expectation is evaluated according to the probability density function (pdf)

$$
f_{\beta}(x)=\left[1-\beta^{-1}\right]^{+} \delta(x)+\frac{\sqrt{\left[x-\eta_{1}\right]^{+}\left[\eta_{2}-x\right]^{+}}}{2 \pi \beta x}
$$

with $[u]^{+} \triangleq \max \{0, u\}$.

2. The spectral efficiency of the CSMU receiver converges almost surely to $C=(\beta / 2) \log (1+\gamma)$.

Proof: To prove part 1 of Theorem 3 we need to determine the limits of $\left[\left(\boldsymbol{R} \boldsymbol{S}^{*} \boldsymbol{S}\right)^{1 / 2}\right]_{m m}^{2}$ and $\zeta[\boldsymbol{R}]_{m m}+\left[\boldsymbol{R} \boldsymbol{S}^{*} \boldsymbol{S}\right]_{m m}$ as $M \rightarrow \infty$ with $\beta$ held constant.

Using the eigendecomposition of $\boldsymbol{S}^{*} \boldsymbol{S}$ and $\boldsymbol{R}$

$$
\left[\left(\boldsymbol{R} \boldsymbol{S}^{*} \boldsymbol{S}\right)^{1 / 2}\right]_{m m}=\left[\boldsymbol{V}(\Sigma \Lambda)^{1 / 2} \boldsymbol{V}^{*}\right]_{m m}=\boldsymbol{v}_{m}^{*}(\Sigma \Lambda)^{1 / 2} \boldsymbol{v}_{m}
$$

where $\boldsymbol{v}_{m}$ is the $m$ th column of $\boldsymbol{V}^{*}$. To determine the limit of (32) when $M \rightarrow \infty$ with $\beta$ held constant, we invoke Lemma 1 below on Wishart matrices which have the form $\boldsymbol{S}^{*} \boldsymbol{S}$ with the elements of $\boldsymbol{S}$ being independent $\mathcal{C N}\left(0, \sigma^{2}\right)$. The lemma relies on the concepts of isotropically distributed vectors and matrices [27].

An $M$-dimensional complex random vector $\phi$ is isotropically distributed if its pdf is invariant to all unitary transformations; i.e., $f(\boldsymbol{\phi})=f\left(\boldsymbol{\Theta}^{*} \boldsymbol{\phi}\right)$ for all $\boldsymbol{\Theta}$ such that $\boldsymbol{\Theta}^{*} \boldsymbol{\Theta}=\boldsymbol{I}_{M}$. If, in addition, $\phi$ is constrained to be a unit vector, then $\phi$ is conveniently generated by $\phi=\boldsymbol{z} / \sqrt{\boldsymbol{z}^{*} \boldsymbol{z}}$, where $\boldsymbol{z}$ is an $M$-dimensional vector of independent $\mathcal{C N}(0,1)$ random variables. An $N \times M$ complex random matrix $\boldsymbol{\Phi}$ is isotropically distributed if its pdf is unchanged when premultiplied by an $M \times M$ unitary matrix; i.e., $f(\boldsymbol{\Phi})=f\left(\boldsymbol{\Theta}^{*} \boldsymbol{\Phi}\right)$ for all $\boldsymbol{\Theta}$ such that $\boldsymbol{\Theta}^{*} \boldsymbol{\Theta}=\boldsymbol{I}_{N}$. The column vectors of $\boldsymbol{\Phi}$ are isotropically distributed vectors.

Lemma 1 ([20], [28]): Let the elements of an $N \times M$ matrix $\boldsymbol{S}$ be independent $\mathcal{C N}\left(0, \sigma^{2}\right)$. Then the eigenvector matrix of $\boldsymbol{S}^{*} \boldsymbol{S}$ is isotropically distributed unitary and independent of the eigenvalues.

From Lemma 1 it follows that $\boldsymbol{v}_{m}$ in (32) is an isotropically distributed unit vector, independent of $\Lambda$ and $\Sigma$. Consequently, $\boldsymbol{v}_{m}$ has the same distribution as $\boldsymbol{z} / \sqrt{\boldsymbol{z}^{*} \boldsymbol{z}}$, where $\boldsymbol{z}$ is an $M$-dimensional vector of independent $\mathcal{C N}(0,1)$ random variables. With $z_{m}$ denoting the components of $\boldsymbol{z}$, it follows that $\left[\left(\boldsymbol{R} \boldsymbol{S}^{*} \boldsymbol{S}\right)^{1 / 2}\right]_{m m}$ has the same distribution as

$$
\frac{\boldsymbol{z}^{*}(\Sigma \Lambda)^{1 / 2} \boldsymbol{z}}{\boldsymbol{z}^{*} \boldsymbol{z}}=\frac{\sum_{m=1}^{M}\left(t\left(\lambda_{m}\right) \lambda_{m}\right)^{1 / 2}\left|z_{m}\right|^{2} / M}{\sum_{m=1}^{M}\left|z_{m}\right|^{2} / M} .
$$

To evaluate the limit of (33) we rely on the following series of lemmas.

Lemma 2 ([29]): If $\lim _{M, N \rightarrow \infty} M / N=\beta \in(0, \infty)$, then the percentage of the $M$ eigenvalues of $\boldsymbol{S}^{*} \boldsymbol{S}$ that lie below $x$ converges to the cumulative distribution function of the pdf $f_{\beta}(x)$ of (31).

Lemma 3: Let $\left\{\boldsymbol{H}_{M}\right\}$ be a sequence of Hermitian matrices, such that $(1 / M) \operatorname{tr}\left(\boldsymbol{H}_{M}\right) \rightarrow q$ as $M \rightarrow \infty$ for some constant $q$, and such that the spectral radius of $\left\{\boldsymbol{H}_{M}\right\}$ is uniformly bounded. Then, with $\boldsymbol{z}_{M}$ denoting a sequence of random vectors with independent and identically distributed (i.i.d.), zero mean, unit variance complex elements with finite eighth moment, we have that ${ }^{2}(1 / M) \boldsymbol{z}_{M}^{*} \boldsymbol{H}_{M} \boldsymbol{z}_{M} \stackrel{\text { a.s. }}{\longrightarrow} q$ as $M \rightarrow \infty$.

Lemma 4: Let $\left\{\boldsymbol{D}_{M}\right\}$ be a sequence of diagonal matrices with diagonal elements $d_{j}=g\left(\lambda_{j}\right)$, where $\lambda_{j}$ is the $j$ th eigenvalue of a Wishart matrix, and $g$ is a function that is continuous on an interval $\mathcal{I}=[a, b]$ where

$$
a<\lambda_{\min } \triangleq(1-\sqrt{\beta})^{2} \quad \text { and } \quad b>\lambda_{\text {max }} \triangleq(1+\sqrt{\beta})^{2} .
$$

Then

$$
\frac{1}{M} \operatorname{tr}\left(\boldsymbol{D}_{M}\right) \stackrel{\text { a.s. }}{\longrightarrow} E\left(g\left(\lambda_{j}\right)\right) .
$$

We now apply Lemmas 3 and 4 to (33). Specifically, we condition on $\Lambda$ and use the fact that $\boldsymbol{z}$ is independent of $\Lambda$. Since, almost surely, $\lambda_{j} \in \mathcal{I}$ for all $M$ large and all $j$ [29], we have

$$
\left[\left(\boldsymbol{R} \boldsymbol{S}^{*} \boldsymbol{S}\right)^{1 / 2}\right]_{m m} \stackrel{\text { a.s. }}{\longrightarrow} E\left\{\left[t\left(\lambda_{1}\right) \lambda_{1}\right]^{1 / 2}\right\}
$$

as $M \rightarrow \infty$, where $E\left\{\left[t\left(\lambda_{1}\right) \lambda_{1}\right]^{1 / 2}\right\}$ is evaluated according to the pdf $f_{\beta}(x)$ of (31). Similarly

$$
\zeta[\boldsymbol{R}]_{m m}+\left[\boldsymbol{R} \boldsymbol{S}^{*} \boldsymbol{S}\right]_{m m} \stackrel{\text { a.s. }}{\longrightarrow} E\left\{t\left(\lambda_{1}\right)\left(\lambda_{1}+\zeta\right)\right\}
$$

which completes the proof of part 1 . The expression for the asymptotic spectral efficiency then follows immediately by noting that $\gamma$ is upper-bounded by the MF single-user bound.

Theorem 3 can be used to determine the asymptotic performance of any linear receiver which can be formulated as a CSMU receiver, with covariance matrix $\boldsymbol{R}$ that satisfies the constraints of the theorem. In Section VI and in [12] and [20], we show that the asymptotic SINR of the MMSE, OMU, and decorrelator receivers, respectively, can be obtained as special cases of Theorem 3.

We have seen in Section IV-B (Proposition 1) that the MF receiver can be formulated as a CSMU receiver with $\boldsymbol{R}=\boldsymbol{S}^{*} \boldsymbol{S}$. Since this choice of $\boldsymbol{R}$ satisfies the conditions of Theorem 3 with $t(\lambda)=\lambda$, the asymptotic SINR is given by

$$
\gamma=\frac{E^{2}\{\lambda\}}{\zeta E\{\lambda\}+E\left\{\lambda^{2}\right\}-E^{2}\{\lambda\}}=\frac{1}{\zeta+\beta}
$$

where we used the fact that $E\{\lambda\}=1[1]$ and $E\left\{\lambda^{2}\right\}=\beta+1$ [30]. The SINR (36) agrees with the asymptotic limit derived in [19], [4].

Similarly, from Proposition 1 it follows that the Müller-Verdú receiver can be formulated as a CSLS receiver with

$$
\begin{aligned}
\boldsymbol{R} & =\left(\sum_{\ell=0}^{L} w_{\ell}\left(\boldsymbol{S}^{*} \boldsymbol{S}\right)^{\ell}\right) \boldsymbol{S}^{*} \boldsymbol{S}\left(\sum_{\ell=0}^{L} w_{\ell}\left(\boldsymbol{S}^{*} \boldsymbol{S}\right)^{\ell}\right) \\
& =\sum_{\ell, k=0}^{L} w_{\ell} w_{k}\left(\boldsymbol{S}^{*} \boldsymbol{S}\right)^{\ell+k+1} .
\end{aligned}
$$

This choice of $R$ also satisfies the conditions of Theorem 3, with

$$
t(\lambda)=\lambda\left(\sum_{\ell=0}^{L} w_{\ell} \lambda^{\ell}\right)^{2} .
$$


From the theorem, the asymptotic SINR is then given by

$$
\gamma=\frac{\left(\sum_{\ell=0}^{L} w_{\ell} E\left\{\lambda^{\ell+1}\right\}\right)^{2}}{\sum_{\ell, k=0}^{L} w_{\ell} w_{k} E\left\{\lambda^{\ell+k+1}(\zeta+\lambda)\right\}}=\frac{\left(\boldsymbol{w}^{*} \boldsymbol{m}\right)^{2}}{\boldsymbol{w}^{*} \Phi \boldsymbol{w}}
$$

where $\boldsymbol{m}$ is a length- $(L+1)$ vector with $\ell$ th element given by $E\left\{\lambda^{\ell}\right\}$, and $\Phi$ is an $(L+1) \times(L+1)$ matrix with $k \ell$ th element given by $E\left\{\lambda^{k+\ell-1}(\lambda+\zeta)\right\}$, where [15]

$$
E\left\{\lambda^{\ell}\right\}=\sum_{m=0}^{\ell-1}\left(\begin{array}{c}
\ell \\
m
\end{array}\right)\left(\begin{array}{c}
\ell \\
m+1
\end{array}\right) \frac{\beta^{m}}{\ell} .
$$

From (39), the weights that maximize the asymptotic SINR are proportional to $\boldsymbol{w}=\Phi^{-1} \boldsymbol{m}$, which agrees with the optimal weights derived in [15].

\section{Example: The MFD Receiver}

We now present an example demonstrating the applicability of Theorem 3.

Suppose we wish to design a linear receiver to operate in a rapidly changing noise environment, so that the SNR changes with time. If the SNR is known at each time instance, then we can design an MMSE receiver matched to the SNR at any given time. However, computing the MMSE receiver at each SNR requires a matrix inverse. To save in computations, we may instead seek a linear receiver whose dependence on the SNR is computationally simple. For example, we may consider a receiver that is a convex combination of the MF and decorrelator receivers, where the coefficient in the combination is chosen to maximize the SINR. Since the MF and decorrelator are both independent of the SNR, the dependence of the resulting receiver on the SNR is only through the scalar coefficient. Specifically, assuming for simplicity that the signatures are linearly independent, we choose a receiver of the form $\hat{x}=Q^{*} r$, where

$$
\boldsymbol{Q}=(1-a) \boldsymbol{S}+a \boldsymbol{S}\left(\boldsymbol{S}^{*} \boldsymbol{S}\right)^{-1}
$$

and $0 \leq a \leq 1$ is chosen to maximize the asymptotic SINR. We refer to this receiver as an MFD (MF + Decorrelator) receiver.

From Proposition 1 it follows that the MFD receiver can be expressed as a CSMU receiver with

$$
\begin{aligned}
\boldsymbol{R} & =\left((1-a) \boldsymbol{S}^{*}+a\left(\boldsymbol{S}^{*} \boldsymbol{S}\right)^{-1} \boldsymbol{S}^{*}\right)\left((1-a) \boldsymbol{S}+a \boldsymbol{S}\left(\boldsymbol{S}^{*} \boldsymbol{S}\right)^{-1}\right) \\
& =(1-a)^{2} \boldsymbol{S}^{*} \boldsymbol{S}+a^{2}\left(\boldsymbol{S}^{*} \boldsymbol{S}\right)^{-1}+2 a(1-a) \boldsymbol{I} .
\end{aligned}
$$

With this choice of $\boldsymbol{R}$, the eigenvalues $t(\lambda)$ are given by

$$
t(\lambda)=(1-a)^{2} \lambda+a^{2} \frac{1}{\lambda}+2 a(1-a) .
$$

To compute the asymptotic SINR using Theorem 3, we first note that we can express $t(\lambda) \lambda$ as

$$
t(\lambda) \lambda=((1-a) \lambda+a)^{2} .
$$

Using the fact that $E\{\lambda\}=1$, we have

$$
E\left\{[t(\lambda) \lambda]^{1 / 2}\right\}=E\{(1-a) \lambda+a\}=1 .
$$

Next, using (43) and the expectations $E\left\{\lambda^{2}\right\}=1+\beta$, and $E\{1 / \lambda\}=1 /(1-\beta)$ for $\beta<1$, we have that

$$
\begin{aligned}
& E\{t(\lambda) \lambda\}=(1-a)^{2}(1+\beta)+a^{2}+2 a(1-a) ; \\
& E\{t(\lambda) \zeta\}=\zeta\left((1-a)^{2}+a^{2} \frac{1}{1-\beta}+2 a(1-a)\right) .
\end{aligned}
$$

The asymptotic SINR of the MFD receiver then follows from Theorem 3 as

$$
\operatorname{SINR}_{\mathrm{MFD}}=\frac{1}{\beta(1-a)^{2}+\zeta\left(1+a^{2} \frac{\beta}{1-\beta}\right)} .
$$

To maximize the SINR we seek the $a$ that is the solution to

$$
\min _{a}\left\{\beta(1-a)^{2}+\zeta\left(1+a^{2} \frac{\beta}{1-\beta}\right)\right\} .
$$

Differentiating with respect to $a$ and equating to 0 , the optimal value of $a$ is

$$
a=\frac{1}{1+\zeta /(1-\beta)} .
$$

Note, that for high SNR, $\zeta \rightarrow 0$, and $a \rightarrow 1$. In this case, the receiver reduces to the decorrelator, as we expect. For low SNR, $\zeta \rightarrow \infty$ and $a \rightarrow 0$, in which case the receiver reduces to the MF.

The asymptotic BER for $\beta=0.8$ using the MFD receiver with the optimal choice of $a$ is illustrated in Fig. 4. For comparison, we also plot the BER resulting from the MMSE, MF, and decorrelator receivers, as well as the Müller-Verdú receiver with $L=1$, which has the same computational cost as the MFD receiver, assuming that the matrix $\left(\boldsymbol{S}^{*} \boldsymbol{S}\right)^{-1}$ is stored in memory, and does not vary with time. The optimal weights for $L=1$ are $w_{0}=\zeta+2(1+\beta)$ and $w_{1}=-1$.

As can be seen from the figure, with the same computational cost, our receiver performs significantly better at high SNR than the Müller-Verdú receiver, with only a negligible loss in performance in the low-SNR regime. We note, however, that our receiver requires storage of the matrix $\left(\boldsymbol{S}^{*} \boldsymbol{S}\right)^{-1}$. If the signature matrix varies with time then the Müller-Verdú receiver will have smaller computational cost since it does not require computation of a matrix inverse.

In the next section we use Theorem 3 to analyze the energy-per-information bit required to achieve a desired spectral efficiency, in the wideband regime, using the CSMU receiver.

\section{Spectral Efficiency in the Wideband Regime}

CDMA systems often operate in the wideband regime where the spectral efficiencies are relatively low [19], [21]. As shown in [22], in this regime the energy-per-information bit, $\frac{E_{b}}{N_{0}}$, required to achieve a desired spectral efficiency, can be approximated up to the first order as

$$
10 \log _{10}\left\{\frac{E_{b}}{N_{0}}(\mathrm{C})\right\} \approx 10 \log _{10}\left\{\frac{E_{b}}{N_{0} \min }\right\}+\frac{\mathrm{C}}{\mathcal{S}_{0}} 10 \log _{10} 2
$$

where $\frac{E_{b}}{N_{0}}$ min denotes the minimum signal energy-per-information bit required for reliable communication, and $\mathcal{S}_{0}$ is the slope of the spectral efficiency curve as a function of $\frac{E_{b}}{N_{0}}$ at $\frac{E_{b}}{N_{0}}$ min, and is referred to as the wideband slope. From (50), it follows that to a first order approximation, $\frac{E_{b}}{N_{\mathrm{omin}}}$ and $\mathcal{S}_{0}$ characterize the required $\frac{E_{b}}{N_{0}}$ of a given system, in the wideband regime.

With $C$ denoting the spectral efficiency, we have that [22]

$$
{\frac{E_{b}}{N_{0} \text { min }}}=\frac{\beta \log _{e} 2}{2 \dot{C}(0)}
$$




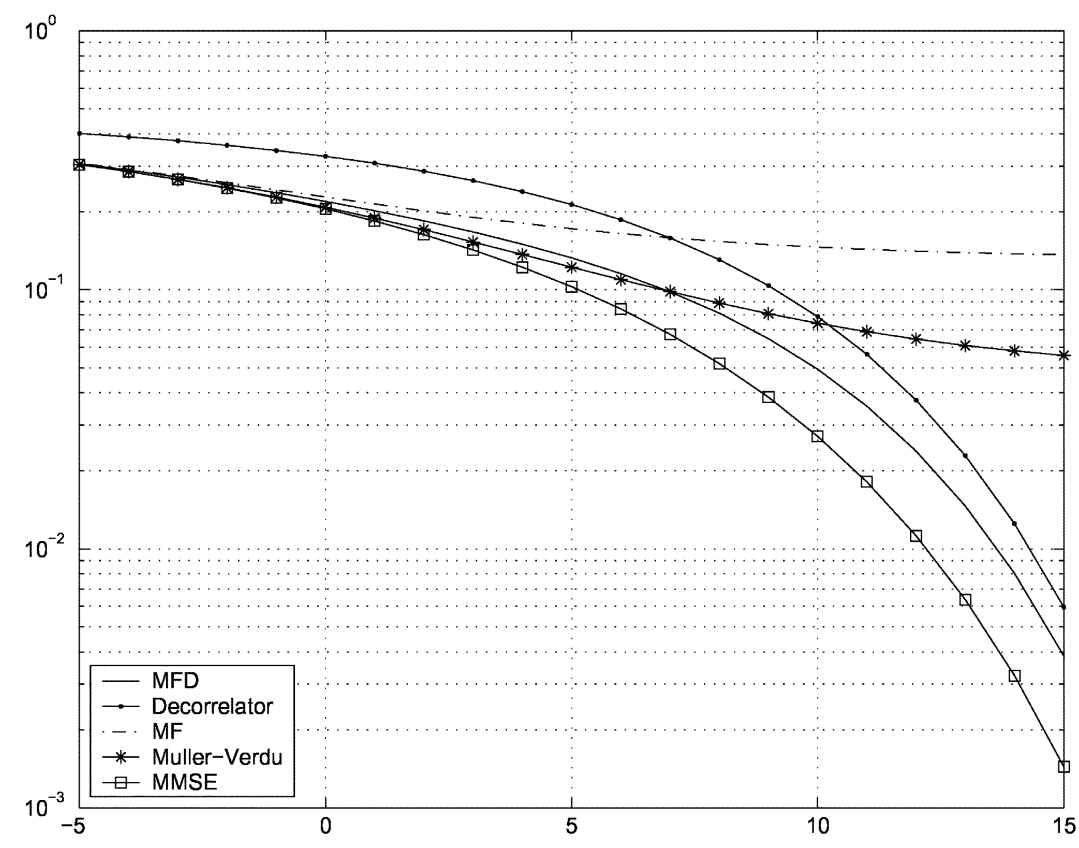

Fig. 4. Probability of bit error as a function of SNR, in the large-system limit, with equal-power users, random signatures, and $\beta=0.8$.

where $\dot{C}(0)$ denotes the derivative of $C$ with respect to $\mathrm{SNR}=1 / \zeta$, evaluated at SNR $=0$. The slope of the spectral efficiency at the point $\frac{E_{b}}{N_{0}}$ min is given by

$$
\mathcal{S}_{0}=-\frac{4 \dot{\mathrm{C}}^{2}(0)}{\ddot{\mathrm{C}}(0)}
$$

where $\ddot{C}(0)$ denotes the second derivative of $C$ with respect to $\mathrm{SNR}$, evaluated at $\mathrm{SNR}=0$.

Theorem 4 below characterizes $\frac{E_{b}}{N_{0} \min }$ and $\mathcal{S}_{0}$ in the large system limit, when using a CSMU receiver.

Theorem 4: Let the elements of the $N \times M$ signature matrix $\boldsymbol{S}$ be independent $\mathcal{C N}(0,1 / N)$, and let the matrix of amplitudes $\boldsymbol{A}=A \boldsymbol{I}$. Let $\boldsymbol{S}^{*} \boldsymbol{S}=\boldsymbol{V} \Lambda \boldsymbol{V}^{*}$ where $\Lambda$ is a diagonal matrix with diagonal elements $\lambda_{m}$, and let $\boldsymbol{R}=\boldsymbol{V} \Sigma \boldsymbol{V}^{*}$ be a covariance matrix with eigenvalues $d_{m}=t\left(\lambda_{m}\right)$ where $t(\cdot)$ is a continuous function on an interval $\mathcal{I}=[a, b]$ where $a<\eta_{1}$ and $b>\eta_{2}$, and $\eta_{1}$ and $\eta_{2}$ are defined by (30). Then in the limit, as $M \rightarrow \infty$ with $\beta \triangleq M / N$ held constant

and

$$
\frac{E_{b}}{N_{0 \text { min }}}=\frac{\log _{e} 2 E\{t(\lambda)\}}{E^{2}\left\{[t(\lambda) \lambda]^{1 / 2}\right\}}
$$

$$
\mathcal{S}_{0}=\frac{2 \beta E^{2}\left\{[t(\lambda) \lambda]^{1 / 2}\right\}}{2 E\{t(\lambda) \lambda\}-E^{2}\left\{[t(\lambda) \lambda]^{1 / 2}\right\}} .
$$

Proof: From Theorem 3, we have that for the CSMU receiver, $\mathrm{C}=(\beta / 2) \log (1+\gamma)$, where $\gamma=\alpha /(1-\alpha)$, and

Therefore,

$$
\alpha=\frac{\operatorname{SNRE}^{2}\left\{[t(\lambda) \lambda]^{1 / 2}\right\}}{E\{t(\lambda)(\lambda \mathrm{SNR}+1)\}}
$$

$$
\dot{\mathrm{C}}=\frac{\beta}{2} \frac{\dot{\alpha}}{1-\alpha}
$$

with

$$
\dot{\alpha}=\frac{E^{2}\left\{[t(\lambda) \lambda]^{1 / 2}\right\} E\{t(\lambda)\}}{E^{2}\{t(\lambda)(1+\lambda \mathrm{SNR})\}}
$$

so that

$$
\dot{\mathrm{C}}(0)=\frac{\beta}{2} \dot{\alpha}(0)=\frac{\beta}{2} \frac{E^{2}\left\{[t(\lambda) \lambda]^{1 / 2}\right\}}{E\{t(\lambda)\}} .
$$

Differentiating (54)

$$
\ddot{\mathrm{C}}=\frac{\beta}{2} \frac{\ddot{\alpha}(1-\alpha)+\dot{\alpha}^{2}}{(1-\alpha)^{2}}
$$

with

$$
\ddot{\alpha}=-2 \frac{E^{2}\left\{[t(\lambda) \lambda]^{1 / 2}\right\} E\{t(\lambda)\} E\{t(\lambda) \lambda\}}{E^{3}\{t(\lambda)(1+\lambda \mathrm{SNR})\}}
$$

so that

$$
\begin{aligned}
\ddot{C}(0) & =\frac{\beta}{2}\left[\ddot{\alpha}(0)+\dot{\alpha}^{2}(0)\right] \\
& =-\frac{\beta}{2} \frac{E^{2}\left\{[t(\lambda) \lambda]^{1 / 2}\right\}\left(2 E\{t(\lambda) \lambda\}-E^{2}\left\{[t(\lambda) \lambda]^{1 / 2}\right\}\right)}{E^{2}\{t(\lambda)\}} .
\end{aligned}
$$

Substituting the expressions for $\dot{C}$ and $\ddot{C}$ into (51) and (52), completes the proof of the theorem.

Since from Theorem 4 , both $\frac{E_{b}}{N_{0}}$ min and $\mathcal{S}_{0}$ are positive, it follows from (50) that to ensure that the energy per bit required for transmission in the wideband regime is minimal, we need $\frac{E_{b}}{N_{0}}$ min to be as small as possible and $\mathcal{S}_{0}$ to be as large as possible.

Using Theorem 4, we can compute $\frac{E_{b}}{N_{0}}$ min and $\mathcal{S}_{0}$ for the MF and decorrelator receivers. For the MF receiver, $t(\lambda)=\lambda$ so that

and

$$
{\frac{E_{b}}{N_{0} \text { min }}}=\log _{e} 2,
$$

$$
\mathcal{S}_{0}=\frac{2 \beta}{1+2 \beta}
$$

where we used that fact that $E\{\lambda\}=1[1]$ and $E\left\{\lambda^{2}\right\}=\beta+1$ [30]. 


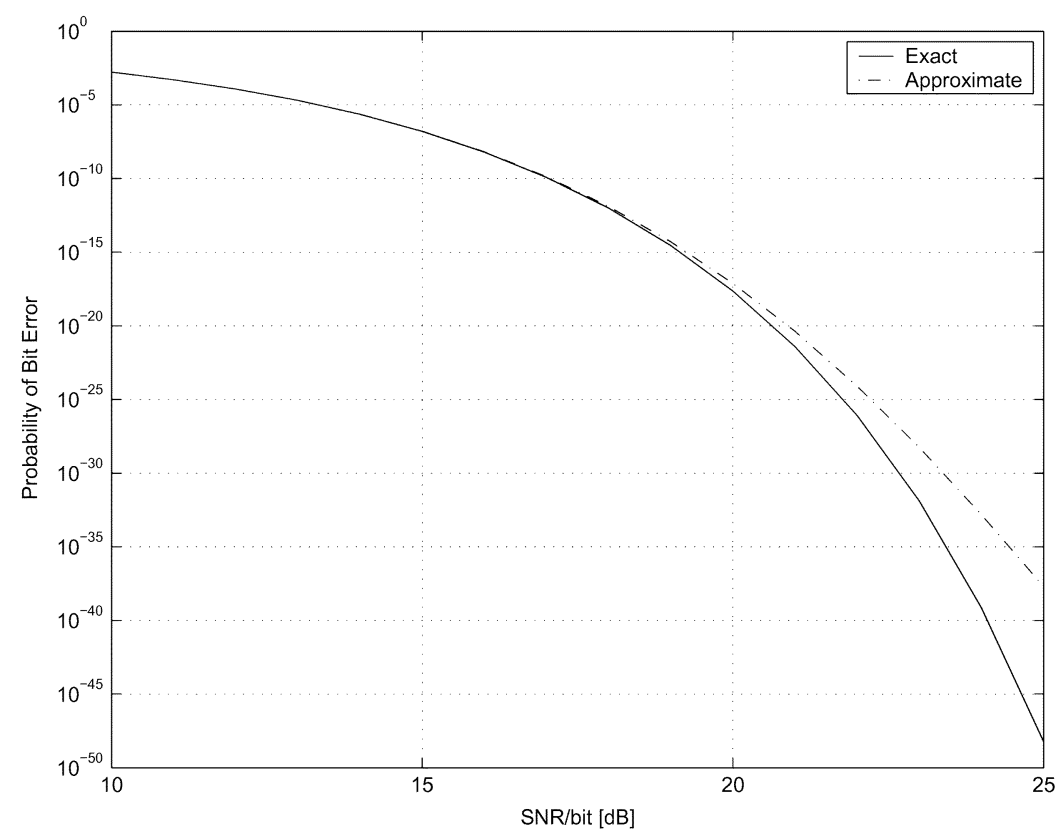

Fig. 5. The exact and Gaussian approximated probability of bit error for the MMSE-based CSMU receiver, with 10 equal-energy users and identical cross correlations $\rho=0.2$.

For the decorrelator receiver with $\beta<1, t(\lambda)=1 / \lambda$ and $E\{1 / \lambda\}=1 /(1-\beta)[1]$. Therefore,

and

$$
\frac{E_{b}}{N_{0} \min }=\frac{\log _{e} 2}{1-\beta}
$$

$$
\mathcal{S}_{0}=2 \beta \text {. }
$$

For $\beta>1$

$$
t(\lambda)= \begin{cases}\frac{1}{\lambda}, & \lambda \neq 0 \\ 0, & \lambda=0 .\end{cases}
$$

It was shown in [20] that $E\{t(\lambda)\}=1 /(\beta(\beta-1))$, and $E\{t(\lambda) \lambda\}=1 / \beta$. Therefore,

and

$$
\frac{E_{b}}{N_{0} \min }=\frac{\beta \log _{e} 2}{\beta-1},
$$

$$
\mathcal{S}_{0}=\frac{2 \beta}{2 \beta-1} .
$$

In the next section, we consider a specific choice of covariance matrix $\boldsymbol{R}$ that does not depend on the channel parameters, and analyze the performance of the resulting CSMU receiver. As we show, over a wide range of the channel parameters the loss in performance using this receiver in comparison with the MMSE receiver is marginal, even though the channel parameters are not known.

\section{MMSE-BASED CSMU RECEIVER}

We now focus on the special case in which $\boldsymbol{R}$ is chosen to have the same form as the output covariance matrix when using the linear MMSE receiver.

In the case of equal power users, the output covariance using the MMSE receiver is $\sigma^{2} \boldsymbol{R}^{\mathrm{MMSE}}$ with

$$
\boldsymbol{R}^{\mathrm{MMSE}}=\left(\boldsymbol{S}^{*} \boldsymbol{S}+\zeta \boldsymbol{I}\right)^{-1} \boldsymbol{S}^{*} \boldsymbol{S}\left(\boldsymbol{S}^{*} \boldsymbol{S}+\zeta \boldsymbol{I}\right)^{-1} .
$$

If the SNR, or equivalently $\zeta$, is not known, then we cannot implement the CSMU receiver with covariance $R^{\mathrm{MMSE}}$. Instead, we propose using a CSMU receiver with output covariance

$$
\boldsymbol{R}=\left(\boldsymbol{S}^{*} \boldsymbol{S}+\delta \boldsymbol{I}\right)^{-1} \boldsymbol{S}^{*} \boldsymbol{S}\left(\boldsymbol{S}^{*} \boldsymbol{S}+\delta \boldsymbol{I}\right)^{-1}
$$

for some parameter $\delta$, where we can think of $1 / \delta$ as an estimate of the SNR. Substituting (68) into (11), the resulting CSMU receiver multiplies $\boldsymbol{r}$ by the matrix $\boldsymbol{Q}^{*}$ with

$$
\boldsymbol{Q}=\boldsymbol{S}\left(\boldsymbol{S}^{*} \boldsymbol{S}+\delta \boldsymbol{I}\right)^{-1} .
$$

The CSMU receiver described by (69) is simply an approximation to the MMSE receiver where the unknown SNR is replaced by an estimate. The advantage of formulating this receiver as a CSMU receiver is that we can now use Theorems 3 and 4 to analyze its performance.

An important issue is how to choose the value of $\delta$ in (69). In Section VI-E, we propose a robust method for choosing $\delta$ in the case in which the SNR changes over a given SNR range. As we show, using the robust MMSE-based receiver, the performance is very close to that of the MMSE receiver over the desired SNR range, so that we almost do not loose anything by not knowing the SNR.

\section{A. Probability of Bit Error}

The exact probability of error using the CSMU receiver of (69) is given by (21), with $\boldsymbol{R}$ given by (68). As discussed in Section V-B, the probability of error can be approximated as $P_{m}(\sigma) \approx \mathcal{Q}(\sqrt{\gamma})$, where $\mathcal{Q}(\cdot)$ is given by (22), and $\gamma$ is given by (28).

In Fig. 5, we compare the accuracy of the Gaussian approximation to the exact BER expression in (21), for the MMSEbased CSMU receiver, with 10 equal-energy users and identical cross correlations $\rho=0.2$. As expected, the approximation is useful at low SNR, but becomes less so at high SNR. 


\section{B. Asymptotic Spectral Efficiency}

The asymptotic SINR of the MMSE-based CSMU receiver given by (69) follows from Theorem 3 , and is given in the following theorem.

Theorem 5: Let the elements of the $N \times M$ signature matrix $\boldsymbol{S}$ be independent $\mathcal{C N}(0,1 / N)$, and let the matrix of amplitudes $\boldsymbol{A}$ be expressible as $A \boldsymbol{I}_{M}$. Then in the limit as $M \rightarrow \infty$ with $\beta \triangleq M / N$ held constant, the SINR for each user at the MMSEbased CSMU receiver output given by (69) is given in (70) at the bottom of the page, where

$$
\mathcal{F}(x, \beta)=\left(\sqrt{x \eta_{2}+1}-\sqrt{x \eta_{1}+1}\right)^{2}
$$

$\eta_{1}$ and $\eta_{2}$ are defined by (30), and $1 / \zeta=A^{2} / \sigma^{2}$ is the received SNR.

Proof: For the covariance matrix $\boldsymbol{R}$ of (68)

$$
t(\lambda)=\frac{\lambda}{(\lambda+\delta)^{2}} .
$$

It therefore follows from Theorem 3 that $\gamma=\alpha /(1-\alpha)$, where

$$
\alpha=\frac{E^{2}\left\{\frac{\lambda}{\lambda+\delta}\right\}}{E\left\{\frac{\lambda(\lambda+\zeta)}{(\lambda+\delta)^{2}}\right\}} .
$$

We first evaluate the numerator of (73). Expressing

$$
E\left\{\frac{\lambda}{\lambda+\delta}\right\}=1-E\left\{\frac{\delta}{\lambda+\delta}\right\}
$$

we have that

$$
E\left\{\frac{\lambda}{\lambda+\delta}\right\}=\frac{1}{4 \beta} \delta \mathcal{F}(1 / \delta, \beta)
$$

where $\mathcal{F}(x, \beta)$ is given by (71). Here we used the identity ([1, p. 303])

$$
E\left\{\frac{\zeta}{\lambda+\zeta}\right\}=1-\frac{1}{4 \beta} \zeta \mathcal{F}(1 / \zeta, \beta)
$$

To evaluate the denominator of (73), we consider separately the expectations $E\left\{\lambda /(\lambda+\delta)^{2}\right\}$ and $E\left\{\lambda^{2} /(\lambda+\delta)^{2}\right\}$. The first expectation can be expressed as

$$
\begin{aligned}
E\left\{\frac{\lambda}{(\lambda+\delta)^{2}}\right\} & =-\frac{d}{d \delta} E\left\{\frac{\lambda}{\lambda+\delta}\right\} \\
& =-\frac{1}{4 \beta}\left(\mathcal{F}(1 / \delta, \beta)+\delta \frac{d}{d \delta} \mathcal{F}(1 / \delta, \beta)\right)
\end{aligned}
$$

where we used (75). Using the fact that

$$
\mathcal{F}(1 / \delta, \beta)=(2 / \delta)\left(1+\beta+\delta-\sqrt{\left(\eta_{1}+\delta\right)\left(\eta_{2}+\delta\right)}\right)
$$

it follows that

$$
\begin{aligned}
\delta \frac{d}{d \delta} \mathcal{F}(1 / \delta, \beta) & \\
= & -\frac{2}{\delta}\left(1+\beta-\sqrt{\left(\eta_{1}+\delta\right)\left(\eta_{2}+\delta\right)}\right) \\
& -\frac{2}{\sqrt{\left(\eta_{1}+\delta\right)\left(\eta_{2}+\delta\right)}}(1+\beta+\delta)
\end{aligned}
$$

$$
\begin{aligned}
& =-\frac{2}{\delta}\left(\frac{\delta \mathcal{F}(1 / \delta, \beta)}{2}-\delta\right)-2 \frac{1+\beta+\delta}{1+\beta+\delta-\delta \mathcal{F}(1 / \delta, \beta) / 2} \\
& =-\mathcal{F}(1 / \delta, \beta)+2\left(1-\frac{1+\beta+\delta}{1+\beta+\delta-\delta \mathcal{F}(1 / \delta, \beta) / 2}\right) .
\end{aligned}
$$

Substituting (78) into (77)

$$
E\left\{\frac{\lambda}{(\lambda+\delta)^{2}}\right\}=\frac{1}{4 \beta} \frac{\delta \mathcal{F}(1 / \delta, \beta)}{1+\beta+\delta-\delta \mathcal{F}(1 / \delta, \beta) / 2} .
$$

To evaluate $E\left\{\lambda^{2} /(\lambda+\delta)^{2}\right\}$, we note that

$$
E\left\{\frac{\lambda^{2}}{(\lambda+\delta)^{2}}\right\}=1-2 \delta E\left\{\frac{\lambda}{(\lambda+\delta)^{2}}\right\}+\delta^{2} E\left\{\frac{1}{(\lambda+\delta)^{2}}\right\}
$$

where

$$
E\left\{\frac{1}{(\lambda+\delta)^{2}}\right\}=-\frac{d}{d \delta} E\left(\frac{1}{\lambda+\delta}\right)
$$

Combining (81), (80), (77), and (76)

$$
\begin{aligned}
E & \left\{\frac{\lambda^{2}}{(\lambda+\delta)^{2}}\right\} \\
& =\frac{\delta}{2 \beta}\left(\mathcal{F}(1 / \delta, \beta)+\delta \frac{d}{d \delta} \mathcal{F}(1 / \delta, \beta)\right)-\frac{\delta^{2}}{4 \beta} \frac{d}{d \delta} \mathcal{F}(1 / \delta, \beta) \\
& =\frac{\delta}{4 \beta}\left(2 \mathcal{F}(1 / \delta, \beta)+\delta \frac{d}{d \delta} \mathcal{F}(1 / \delta, \beta)\right) \\
& =\frac{\delta \mathcal{F}(1 / \delta, \beta)}{4 \beta}\left(1-\frac{\delta}{1+\beta+\delta-\delta \mathcal{F}(1 / \delta, \beta) / 2}\right)
\end{aligned}
$$

and

$$
\begin{aligned}
E & \left\{\frac{\lambda(\lambda+\zeta)}{(\lambda+\delta)^{2}}\right\} \\
& =\frac{\delta \mathcal{F}(1 / \delta, \beta)}{4 \beta}\left(1+\frac{\zeta-\delta}{1+\beta+\delta-\frac{1}{2} \delta \mathcal{F}(1 / \delta, \beta)}\right) .
\end{aligned}
$$

Therefore,

$$
\alpha=\frac{\delta \mathcal{F}(1 / \delta, \beta)}{4 \beta}\left(1+\frac{\delta-\zeta}{1+\beta+\zeta-\frac{1}{2} \delta \mathcal{F}(1 / \delta, \beta)}\right)
$$

and the asymptotic SINR is given in (85) at the bottom of the following page. Using the identity ([1, p. 304])

$$
1-\frac{\delta \mathcal{F}(1 / \delta, \beta)}{4 \beta}=\left(1+\frac{1}{\delta}-\frac{1}{4} \mathcal{F}(1 / \delta, \beta)\right)^{-1}
$$

we have that

$$
\begin{aligned}
\frac{4 \beta}{\delta \mathcal{F}(1 / \delta, \beta)}-1 & =\left(\frac{1}{1-\frac{\delta \mathcal{F}(1 / \delta, \beta)}{4 \beta}}-1\right)^{-1} \\
& =\left(\frac{1}{\delta}-\frac{1}{4} \mathcal{F}(1 / \delta, \beta)\right)^{-1}
\end{aligned}
$$

and $\gamma$ reduces to (70) at the bottom of the page, completing the proof of the theorem.

$$
\gamma=\frac{1+\beta+\delta-\frac{1}{2} \delta \mathcal{F}(1 / \delta, \beta)}{\left(\frac{1}{\delta}-\frac{1}{4} \mathcal{F}(1 / \delta, \beta)\right)^{-1}\left(1+\beta+\zeta-\frac{1}{2} \delta \mathcal{F}(1 / \delta, \beta)\right)+\zeta-\delta}
$$




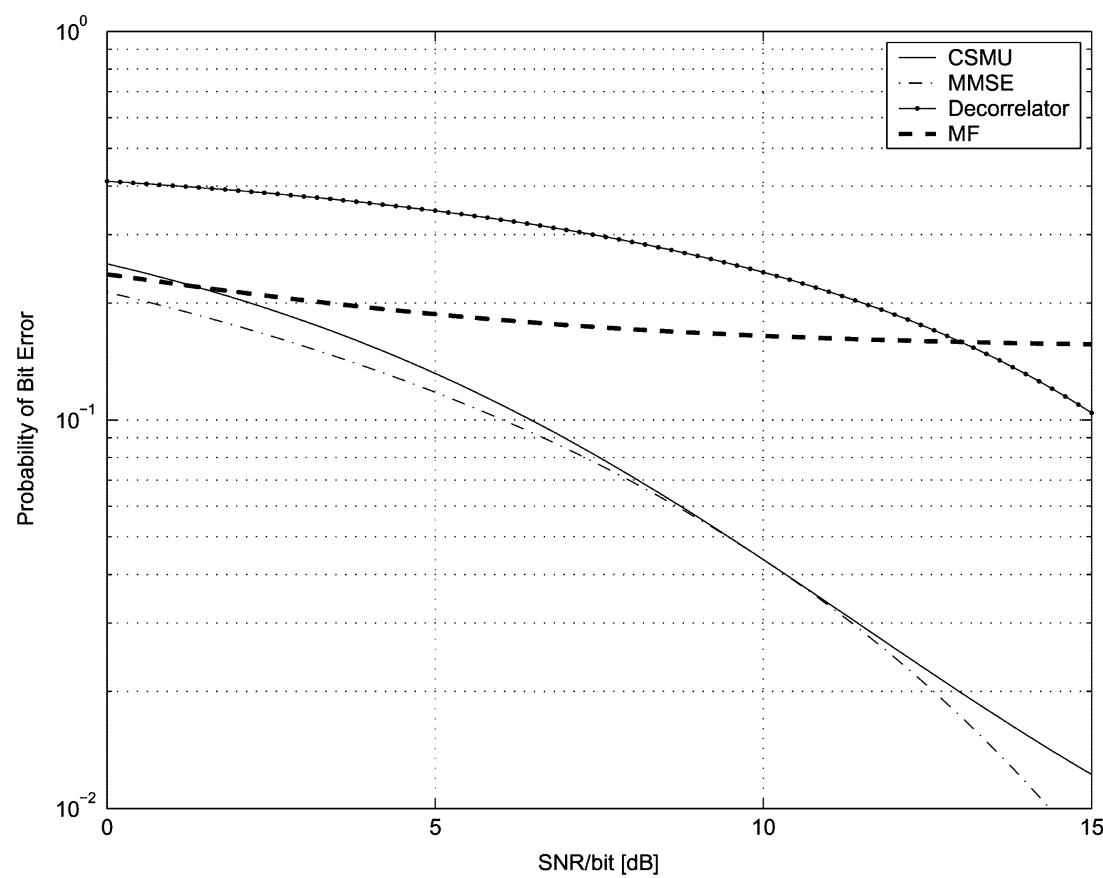

Fig. 6. Probability of bit error as a function of SNR, in the large-system limit, with equal-power users, random signatures, and $\beta=0.95$. The CSMU receiver is given by (69) with $1 / \delta=10[\mathrm{~dB}]$.

Since the MAI is asymptotically Gaussian in the infinite-user limit [31], we expect the Gaussian approximation (29) to be an accurate approximation to the bit-error rate at all SNR, where $\gamma$ is given by Theorem 4 . We will use this approximation to compute the bit-error rate for the remainder of this section.

In Fig. 6, the BER in the infinite-user limit for the MMSEbased CSMU receiver with $1 / \delta=10[\mathrm{~dB}]$, is compared to the $\mathrm{MF}$, the decorrelator, and the MMSE receiver, for $\beta=0.95$. For the SNR range shown, the CSMU receiver performs better than the decorrelator and the MF, and its performance is close to that of the MMSE receiver.

In Fig. 7, we plot the probability of bit error ${ }^{3}$ in the infiniteuser limit as a function of $\beta$, with $1 / \zeta=6[\mathrm{~dB}]$ and $1 / \delta=$ $10[\mathrm{~dB}]$. As can be seen from the figure, the performance of the MMSE-based CSMU receiver is very close to that of the MMSE receiver.

\section{Spectral Efficiency}

From Theorem 5, the spectral efficiency of the CSMU receiver of (69) converges almost surely as $M \rightarrow \infty$ to (88) at the bottom of the page.

${ }^{3}$ The asymptotic large system performance of the decorrelator for the case $\beta>1$ is derived in [20].
We now consider the loss in spectral efficiency using the CSMU receiver of (69), which does not rely on knowledge of the channel parameters, with respect to the MMSE receiver, which assumes knowledge of these parameters.

The spectral efficiency of the MMSE receiver in the large system limit $\lim _{M \rightarrow \infty} C^{\mathrm{MMSE}}$ follows from (88) with $\delta=\zeta$, and is given by

$$
\lim _{M \rightarrow \infty} \mathrm{C}^{\mathrm{MMSE}}=\frac{\beta}{2} \log \left(1+\frac{1}{\zeta}-\frac{1}{4} \mathcal{F}(1 / \zeta, \beta)\right) .
$$

Note that this expression agrees with the asymptotic limit derived in [19].

From (88) and (89), the loss in spectral efficiency using the CSMU receiver of (69) with respect to the MMSE receiver is

$$
\Delta \mathrm{C}=\lim _{M \rightarrow \infty}\left(\mathrm{C}^{\mathrm{MMSE}}-\mathrm{C}\right)=-\frac{\beta}{2} \log (T(\delta, \zeta, \beta))
$$

where we define

$$
T(\delta, \zeta, \beta)=\frac{H(\delta, \beta)+\zeta}{(H(\delta, \beta)+\delta) \frac{G(\zeta, \beta)}{G(\delta, \beta)}+(\zeta-\delta) G(\zeta, \beta)} .
$$

Here

$$
G(\zeta, \beta)=1+\frac{1}{\zeta}-\frac{1}{4} \mathcal{F}(1 / \zeta, \beta)
$$

$$
\gamma=\frac{1+\beta+\delta-\frac{1}{2} \delta \mathcal{F}(1 / \delta, \beta)}{\left(1+\beta+\delta-\frac{1}{2} \delta \mathcal{F}(1 / \delta, \beta)\right)\left(\frac{4 \beta}{\delta \mathcal{F}(1 / \delta, \beta)}-1\right)+(\zeta-\delta) \frac{4 \beta}{\delta \mathcal{F}(1 / \delta, \beta)}} .
$$

$$
\lim _{M \rightarrow \infty} \mathrm{C}=\frac{\beta}{2} \log (1+\gamma)=\frac{\beta}{2} \log \left(\frac{1+\beta+\zeta-\frac{1}{2} \delta \mathcal{F}(1 / \delta, \beta)}{\left(1+\beta+\delta-\frac{1}{2} \delta \mathcal{F}(1 / \delta, \beta)\right)\left(1+\frac{1}{\delta}-\frac{1}{4} \mathcal{F}(1 / \delta, \beta)\right)^{-1}+\zeta-\delta}\right)
$$




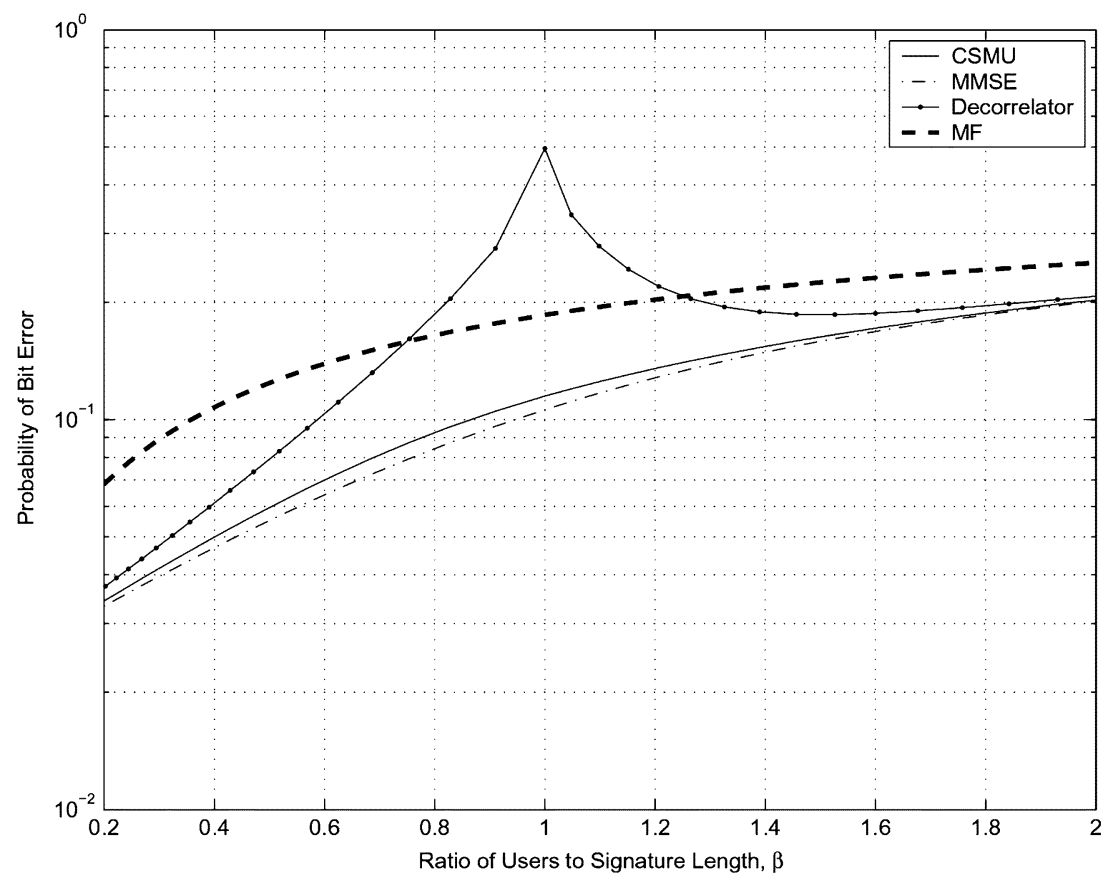

Fig. 7. Probability of bit error as a function of $\beta$ in the large-system limit, with equal-power users, random signatures, and $1 / \zeta=6[\mathrm{~dB}]$. The CSMU receiver is given by (69) with $1 / \delta=10[\mathrm{~dB}]$.

and

$$
H(\delta, \beta)=1+\beta-\frac{1}{2} \delta \mathcal{F}(1 / \delta, \beta)
$$

To analyze the loss in spectral efficiency using the CSMU receiver of (69), we examine the behavior of $\Delta C$ for different values of the parameters $\zeta, \delta$, and $\beta$. In our development, when we refer to the CSMU receiver we explicitly mean the CSMU receiver of (69).

1) Fixed $\zeta$ and $\delta$ : We first consider the behavior of $\Delta C$ as a function of $\beta$, for fixed $\zeta$ and $\delta$.

For fixed $\delta$ we have that

$$
\mathcal{F}(1 / \delta, \beta) \rightarrow \begin{cases}\frac{4}{\delta}, & \beta \gg 1 \\ 0, & \beta \ll 1\end{cases}
$$

Therefore, from (93)

$$
H(\delta, \beta) \rightarrow \begin{cases}\beta, & \beta \gg 1 \\ 1, & \beta \ll 1\end{cases}
$$

and from (92)

$$
G(\delta, \beta) \rightarrow \begin{cases}1, & \beta \gg 1 \\ 1+\frac{1}{\delta}, & \beta \ll 1 .\end{cases}
$$

Substituting (95) and (96) into the expression for $T(\delta, \zeta, \beta)$ of (91)

$$
T(\delta, \zeta, \beta) \rightarrow 1, \quad \beta \ll 1 \quad \text { or } \quad \beta \gg 1
$$

We conclude that for fixed $\zeta$ and $\delta, \Delta \mathrm{C}=0$ for high and low values of $\beta$. The spectral efficiency of the CSMU and MMSE receivers as a function of $\beta$ are illustrated in Fig. 8 , for $1 / \zeta=$ $10[\mathrm{~dB}]$ and $1 / \delta=7[\mathrm{~dB}]$. As is evident from the figure, for fixed SNR the loss in capacity using the CSMU receiver of (69) for all values of $\beta$ is very small.

2) Fixed $\beta$ : We now discuss the behavior of $\Delta C$ for fixed $\beta$ in the limits of high and low SNR.

For low SNR values, $\zeta \gg 1, \zeta \mathcal{F}(1 / \zeta, \beta) \rightarrow 0$, and for high SNR values, $\zeta \ll 1$

$$
\zeta \mathcal{F}(1 / \zeta, \beta) \rightarrow 2\left(1+\beta-\sqrt{\eta_{2} \eta_{1}}\right)= \begin{cases}4 \beta, & \beta \leq 1 \\ 4, & \beta \geq 1\end{cases}
$$

From (93) and (92) we then have that for $\zeta, \delta \gg 1, H(\delta, \beta) \rightarrow$ $1+\beta$ and $G(\zeta, \beta) \rightarrow 1$. For $\zeta, \delta \ll 1$

$$
H(\delta, \beta) \rightarrow \begin{cases}1-\beta, & \beta \leq 1 \\ \beta-1, & \beta \geq 1\end{cases}
$$

and

$$
G(\zeta, \beta) \rightarrow \begin{cases}\infty, & \beta \leq 1 \\ \frac{\beta}{\beta-1}, & \beta \geq 1\end{cases}
$$

We also have that for $\zeta, \delta \ll 1$

$$
\frac{G(\zeta, \beta)}{G(\delta, \beta)}=\frac{\zeta G(\zeta, \beta)}{\zeta G(\delta, \beta)} \rightarrow \frac{\delta}{\zeta} .
$$

Using these limits

$$
T(\delta, \zeta, \beta) \rightarrow 1, \quad \zeta, \delta \ll 1, \quad \text { or } \quad \zeta, \delta \gg 1
$$

and $\Delta \mathrm{C} \rightarrow 0$. Thus, for fixed $\beta$, when $\delta, \zeta \gg 1$ or $\delta, \zeta \ll$ $1, \Delta \mathrm{C}=0$ so that there is no loss in spectral efficiency using the CSMU receiver in place of the MMSE receiver.

3) Fixed $\beta$ and $\delta$ : We now treat the case in which both $\beta$ and $\delta$ are fixed.

For low $\operatorname{SNR} \zeta \gg 1, T(\delta, \zeta, \beta) \rightarrow 1$, and $\Delta \mathrm{C} \rightarrow 0$. 


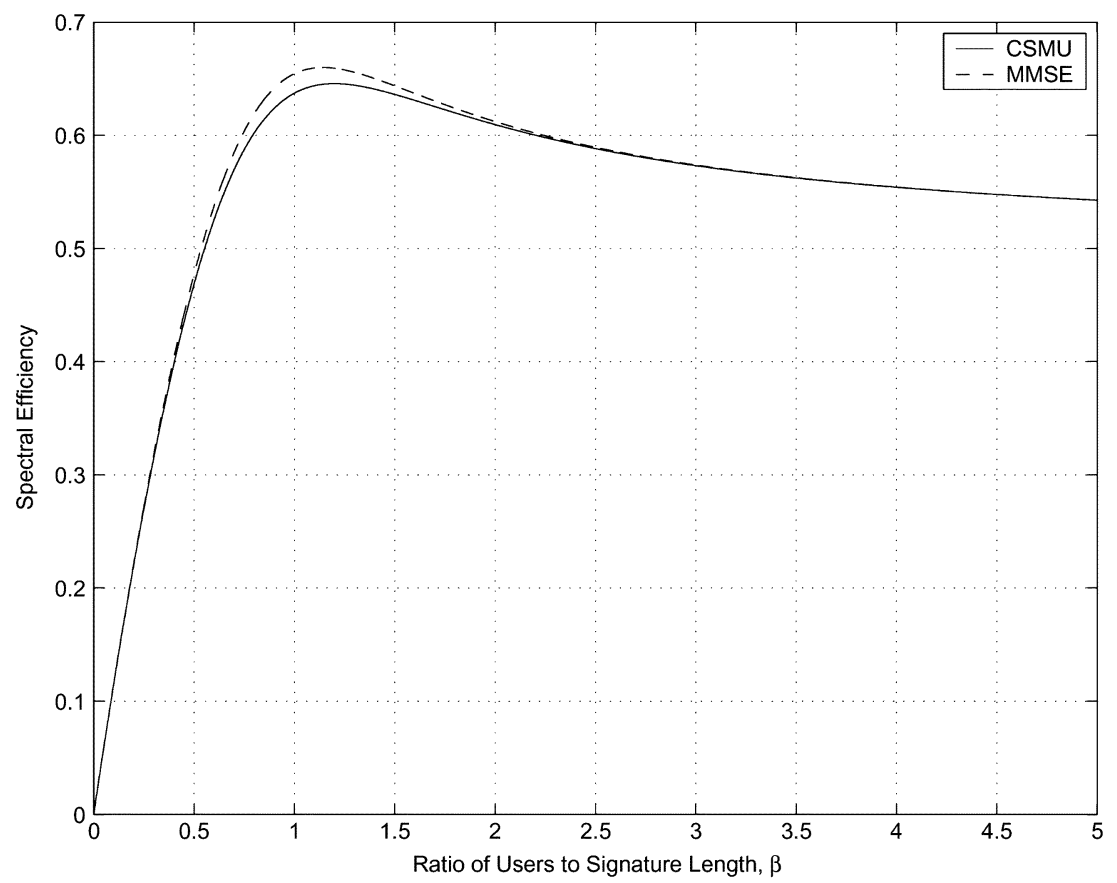

Fig. 8. Spectral efficiency of the CSMU and MMSE receivers as a function of $\beta$, for $1 / \zeta=10[\mathrm{~dB}]$ and $1 / \delta=7[\mathrm{~dB}]$.

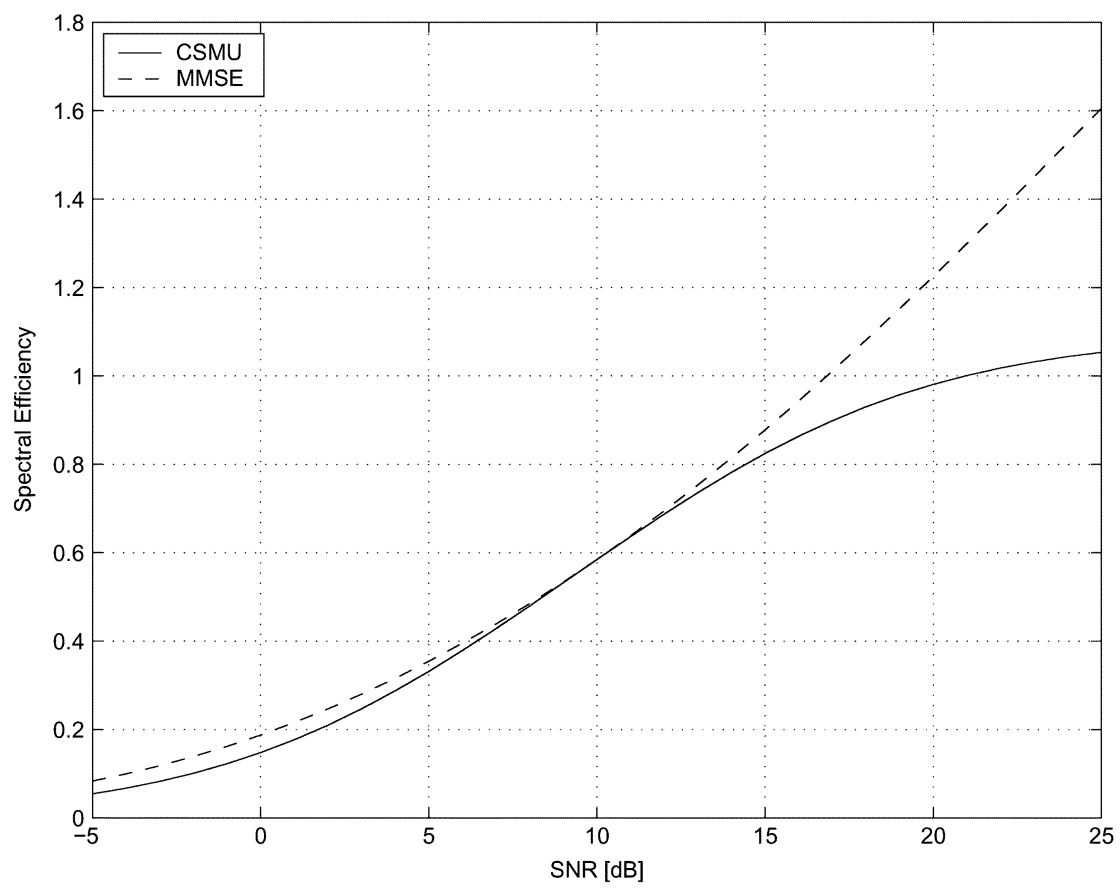

Fig. 9. Spectral efficiency of the CSMU and MMSE receivers as a function of SNR, for $\beta=0.7$ and $1 / \delta=10$ [dB].

Next, suppose that $\zeta \ll 1$. For $\beta \leq 1, T(\delta, \zeta, \beta) \rightarrow 0$ and $\Delta C \rightarrow-\infty$. For $\beta>1$

$$
T(\delta, \zeta, \beta) \rightarrow \frac{H(\delta, \beta)}{\left(\frac{H(\delta, \beta)+\delta}{G(\delta, \beta)}-\delta\right) \frac{\beta}{\beta-1}} \triangleq T^{*}
$$

and

$$
\Delta \mathrm{C}^{*}=-\beta / 2 \log \left(T^{*}\right) .
$$

We conclude that if $\beta$ and $\delta$ are fixed, then for low to intermediate SNR $\Delta C \rightarrow 0$ so that there is no loss in spectral efficiency. For high SNR, if $\beta<1$ then $\Delta C$ grows without bound as a function of SNR. This is because in this regime, the spectral efficiency of the CSMU receiver tends to a limit while the spectral efficiency of the MMSE receiver grows with increasing SNR, as can be seen in Fig. 9. Note, however, from Fig. 9 that for low to intermediate SNR values, the loss in spectral efficiency is very small.

If $\beta>1$, then for high SNR $\Delta C$ converges to the limit given by (103) and (104). As we show, this limit is typically small. This can also be seen in Fig. 10 in which we plot the spectral efficiency of the CSMU and MMSE receivers as a function of SNR, for $\beta=3$ and $1 / \delta=10[\mathrm{~dB}]$. 


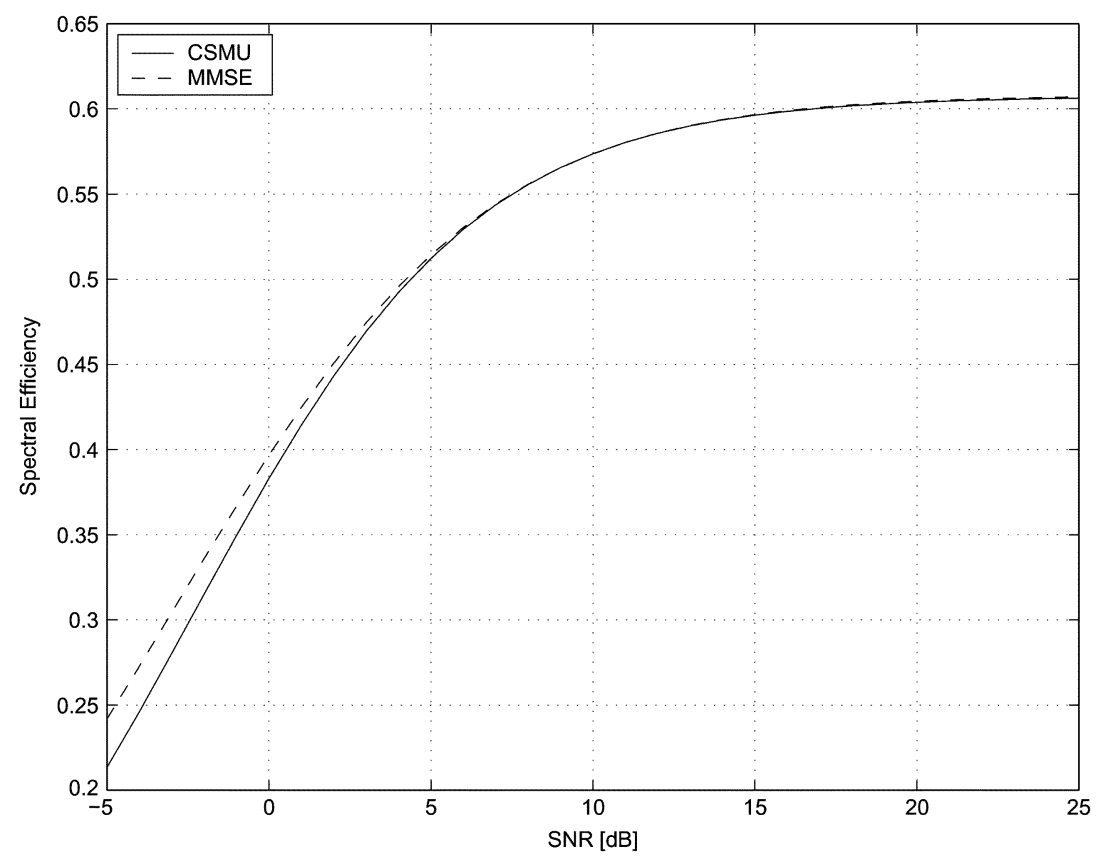

Fig. 10. Spectral efficiency of the CSMU and MMSE receivers as a function of SNR, for $\beta=3$ and $1 / \delta=10$ [dB].

We now consider the high SNR limit (103) for different values of $\delta$ and fixed $\beta>1$. For $\delta \ll 1, H(\delta, \beta) \rightarrow \beta-1$, and $G(\delta, \beta) \rightarrow \beta /(\beta-1)$ so that

$$
T^{*} \rightarrow \frac{\beta(\beta-1)}{\beta(\beta-1)}=1
$$

and $\Delta \mathrm{C}^{*}=0$. For $\delta \gg 1, H(\delta, \beta) \rightarrow 1+\beta, G(\delta, \beta) \rightarrow 1$ and $\delta(1-G(\delta)) \rightarrow-1$. Therefore,

$$
T^{*} \rightarrow \frac{(\beta-1)(1+\beta)}{\beta^{2}}=1-\frac{1}{\beta^{2}}
$$

and $\Delta C^{*}=-\beta / 2 \log \left(T^{*}\right)$. The behavior of $\Delta C^{*}$ as a function of $1 / \delta$ is illustrated in Fig. 11. As can be seen from the figure, this limiting difference is very small so that for typical values of $\delta$, there is essentially no loss of capacity.

For fixed $\delta$ and $\beta \gg 1, H(\delta, \beta) \rightarrow \beta, \mathcal{F}(1 / \delta, \beta) \rightarrow 4 / \delta$, and $G(\delta, \beta) \rightarrow 1$ so that

$$
T^{*} \rightarrow \frac{\beta-1}{\beta} \rightarrow 1
$$

and $\Delta \mathrm{C}^{*} \rightarrow 0$.

To summerize, for fixed $\beta$, when both $\delta$ and $\zeta$ are much greater or much smaller than 1 , then there is no loss in spectral efficiency. When both $\delta$ and $\beta$ are fixed, then for low to intermediate values of SNR there is almost no loss in spectral efficiency. For high SNR values, if $1 / \delta$ is large or $\beta>1$, then again there is essentially no loss in spectral efficiency, as can be seen in Fig. 10. For $\beta<1$, the loss in spectral efficiency over a wide range of SNR values will be small if we choose $\delta$ to be small. In particular, we can always choose $\delta$ so that the loss in spectral efficiency with respect to the MMSE receiver over an SNR range of interest is small, as illustrated in Fig. 12. In some cases, this will entail a larger loss in other SNR regimes. However, it seems reasonable that although the receiver may be operating in a changing environment, so that the SNR will fluctuate, there is a range of SNR values over which fluctuations will occur. Over this range, the parameters can be chosen to achieve essentially the same capacity as the MMSE receiver.

\section{Wideband Regime}

We now consider the energy-per-information bit required in the wideband regime as a function of the spectral efficiency using the MMSE-based CSMU receiver, and compare it with that of the MMSE receiver.

We have seen in Section V-D that energy-per-information bit is characterized by $\frac{E_{b}}{N_{0}}$ min and the wideband slope $\mathcal{S}_{0}$, which for the CSMU receiver, are given by Theorem 4 . We now calculate these expressions for the special case of the MMSE-based CSMU receiver, in which $t(\lambda)$ is given by (72). tions

To evaluate $\frac{E_{b}}{N_{0} \min }$ and $\mathcal{S}_{0}$ we need to compute the expecta-

$$
\begin{aligned}
E\{t(\lambda)\} & =E\left\{\frac{\lambda}{(\lambda+\delta)^{2}}\right\} \\
E\left\{[t(\lambda) \lambda]^{1 / 2}\right\} & =E\left\{\frac{\lambda}{\lambda+\delta}\right\} \\
E\{t(\lambda) \lambda\} & =E\left\{\frac{\lambda^{2}}{(\lambda+\delta)^{2}}\right\}
\end{aligned}
$$

which are given by (79), (75), and (82), respectively. From Theorem 4 we then have that $\frac{E_{b}}{N_{0}}$ min and $\mathcal{S}_{0}$ for the MMSE-based CSMU receiver of (69) are given by

$$
\frac{E_{b}}{N_{0 \min }}=\frac{4 \beta \log _{e} 2}{\delta \mathcal{F}(1 / \delta, \beta)(1+\beta+\delta-\delta \mathcal{F}(1 / \delta, \beta) / 2)}
$$
and

$$
\mathcal{S}_{0}=\frac{2 \beta}{\frac{8 \beta}{\delta \mathcal{F}(1 / \delta, \beta)}\left(1-\frac{\delta}{1+\beta+\delta-\delta \mathcal{F}(1 / \delta, \beta) / 2}\right)-1}
$$




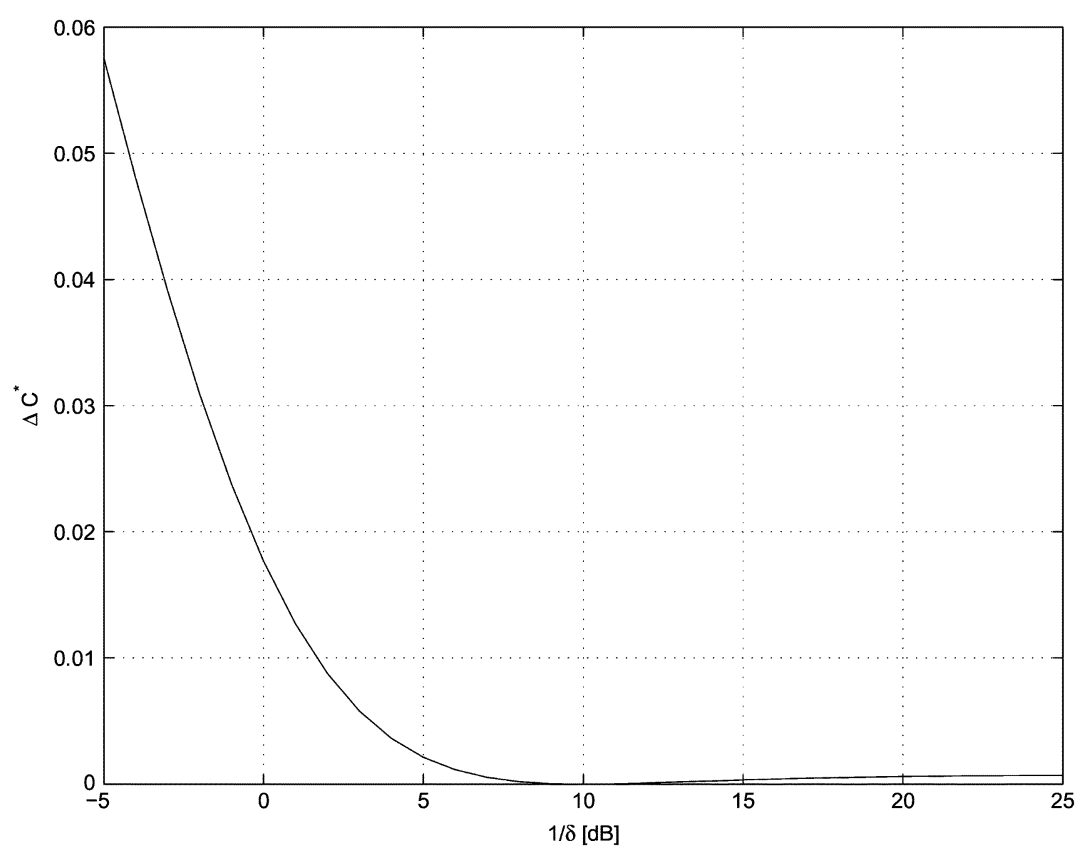

Fig. 11. $\Delta \mathrm{C}^{*}$ of (104) as a function of $1 / \delta$ for $\beta=3$.

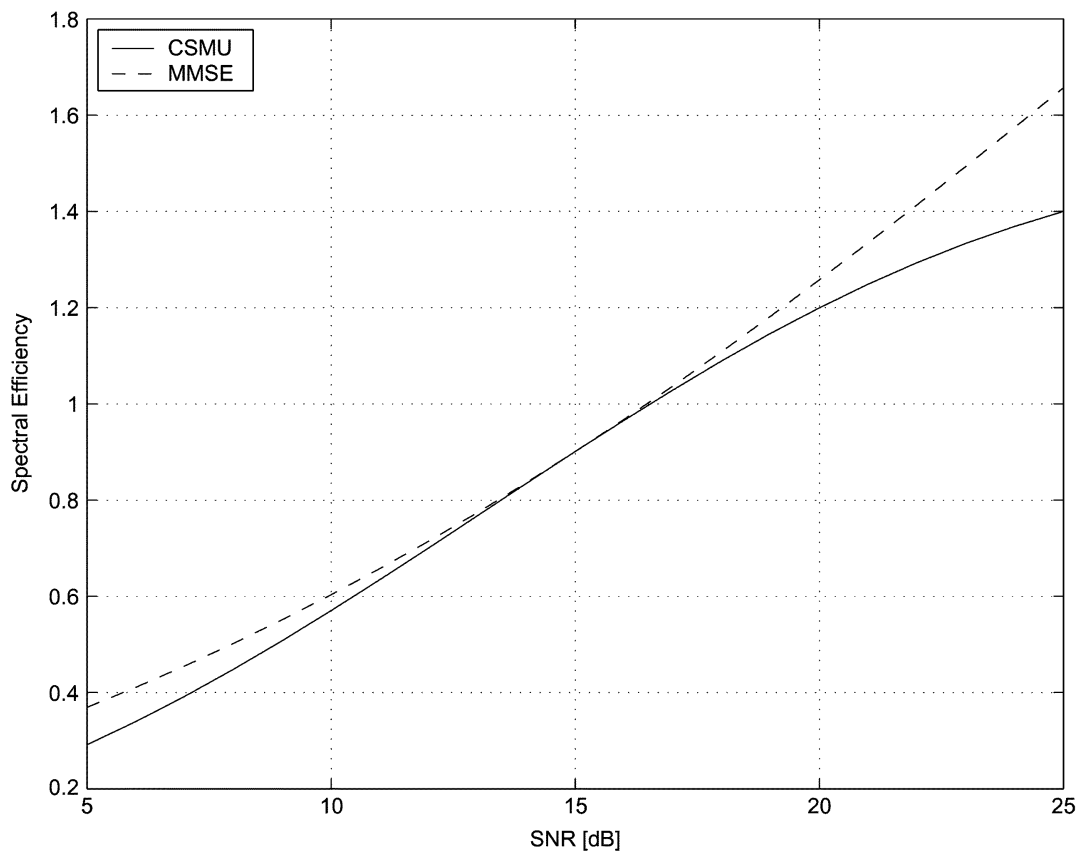

Fig. 12. Spectral efficiency of the CSMU and MMSE receivers for $\beta=0.75$ and $1 / \delta=15$ [dB].

respectively. We now compare these expressions with the $\frac{E_{b}}{N_{0}} \min$ and $\mathcal{S}_{0}$ when using the MMSE receiver.

For the MMSE receiver, $C=(\beta / 2) \log (1+\gamma)$ where

$$
\gamma=\mathrm{SNR}-\frac{1}{4} \mathcal{F}(\mathrm{SNR}, \beta)
$$

so that

$$
\dot{\mathrm{C}}=\frac{\beta}{2} \frac{1}{1+\gamma}\left(1-\frac{1}{4} \frac{d}{d \mathrm{SNR}} \mathcal{F}(\mathrm{SNR}, \beta)\right) .
$$

It is easy to see that for SNR $=0$, both the derivative of $\mathcal{F}(\mathrm{SNR}, \beta)$ and $\gamma$ are equal to zero. Therefore, $\dot{\mathrm{C}}(0)=\beta / 2$ and

$$
{\frac{E_{b}}{N_{0} \min }}^{\mathrm{MMSE}}=\log _{e} 2 .
$$

Differentiating (112) with respect to SNR

$$
\begin{aligned}
\ddot{\mathrm{C}}=-\frac{\beta}{2} \frac{1}{(1+\gamma)^{2}}(1- & \left.\frac{1}{4} \frac{d}{d \operatorname{SNR}} \mathcal{F}(\mathrm{SNR}, \beta)\right)^{2} \\
& -\frac{\beta}{8} \frac{1}{1+\gamma} \frac{d^{2}}{d \mathrm{SNR}^{2}} \mathcal{F}(\mathrm{SNR}, \beta)
\end{aligned}
$$




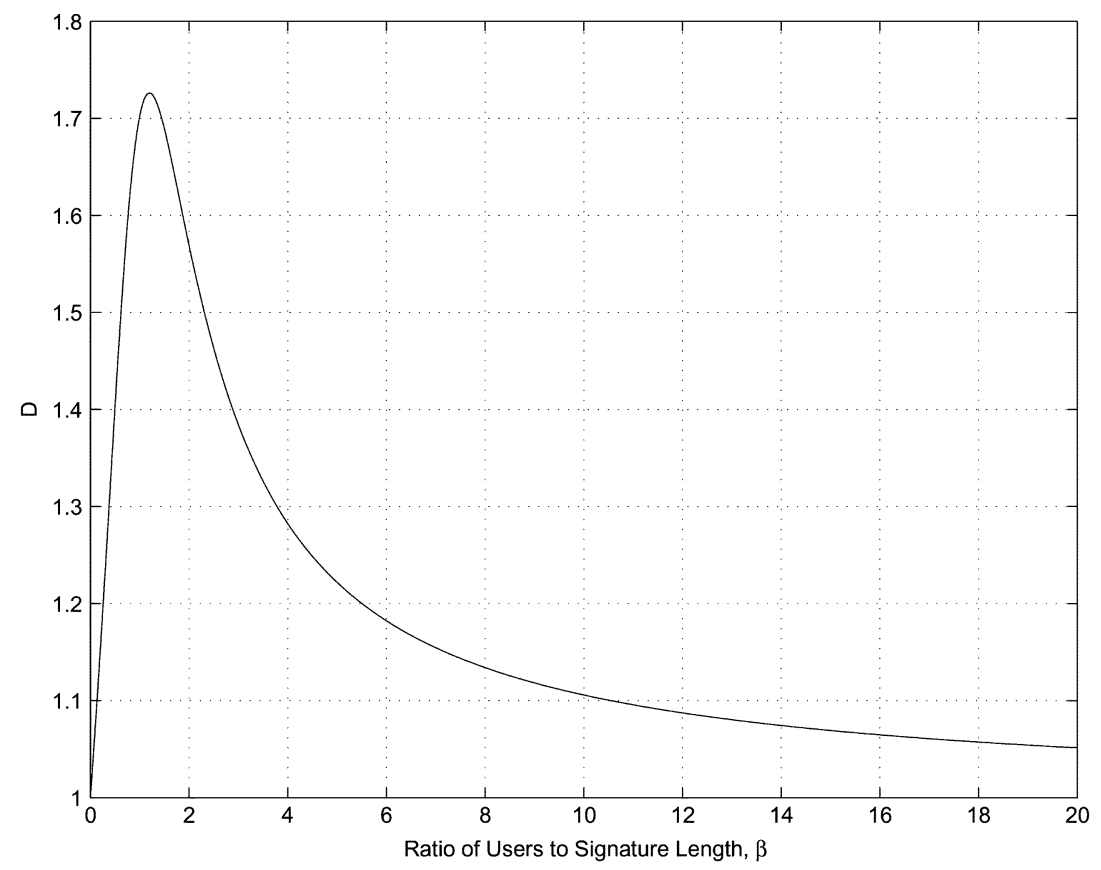

Fig. 13. $\mathcal{D}(\delta, \beta)$ of (117) as a function of $\beta$, for $1 / \delta=7[\mathrm{~dB}]$.

where it can be shown that for SNR $=0$

$$
d^{2} / d \operatorname{SNR}^{2}(\mathcal{F}(\operatorname{SNR}, \beta))=8 \beta
$$

so that $\ddot{C}(0)=-\beta / 2-\beta^{2}$, which results in

$$
\mathcal{S}_{0}^{\mathrm{MMSE}}=\frac{2 \beta}{1+2 \beta} .
$$

Combining (109) with (113), we conclude that $\frac{E_{b}}{N_{0}}$ min of the MMSE-based CSMU receiver can be expressed as

$$
{\frac{E_{b}}{N_{0} \min }}_{N_{0 \min }}={\frac{E_{b}}{N_{M M S E}}}^{\mathrm{D}(\delta, \beta)}
$$

where

$$
\mathcal{D}(\delta, \beta) \triangleq \frac{4 \beta}{\delta \mathcal{F}(1 / \delta, \beta)(1+\beta+\delta-\delta \mathcal{F}(1 / \delta, \beta) / 2)} .
$$

The function $\mathcal{D}(\delta, \beta)$ captures the loss in $\frac{E_{b}}{N_{0}}$ using the MMSEbased CSMU receiver, with respect to the MMSE receiver.

Similarly, combining (110) with (115), $\mathcal{S}_{0}$ of the MMSEbased CSMU receiver can be expressed as

$$
\mathcal{S}_{0}=\mathcal{S}_{0}^{\mathrm{MMSE}} \mathcal{G}(\delta, \beta)
$$

where

$$
\mathcal{G}(\delta, \beta) \triangleq \frac{1+2 \beta}{\frac{8 \beta}{\delta \mathcal{F}(1 / \delta, \beta)}\left(1-\frac{\delta}{1+\beta+\delta-\delta \mathcal{F}(1 / \delta, \beta) / 2}\right)-1}
$$

and captures the loss in the wideband slope using the MMSEbased CSMU receiver, with respect to the MMSE receiver.

Ideally, we would like $\mathcal{D}(\delta, \beta)$ and $\mathcal{G}(\delta, \beta)$ to be close to 1 . As we now show, for certain choices of the parameters $\delta$ and $\beta, \mathcal{D}(\delta, \beta) \rightarrow 1$ and $\mathcal{G}(\delta, \beta) \rightarrow 1$, so that in these cases there is essentially no loss in $\frac{E_{b}}{N_{0}}$ with respect to the MMSE receiver.

To this end, we analyze the behavior of $\mathcal{D}(\delta, \beta)$ and $\mathcal{G}(\delta, \beta)$ for different values of $\delta$ and $\beta$.
For fixed $\delta$

$$
\delta \mathcal{F}(1 / \delta, \beta) \rightarrow \begin{cases}4, & \beta \gg 1 \\ 0, & \beta \ll 1\end{cases}
$$

so that

$$
\mathcal{D}(\delta, \beta) \rightarrow 1, \quad \beta \gg 1, \quad \beta \ll 1
$$

The function $\mathcal{D}(\delta, \beta)$ as a function of $\beta$ is illustrated in Fig. 13, for $1 / \delta=10[\mathrm{~dB}]$.

Similarly, from (120) it follows that

$$
\mathcal{G}(\delta, \beta) \rightarrow 1, \quad \beta \gg 1 .
$$

For $\beta \ll 1, \mathcal{S}_{0}^{\mathrm{MMSE}} \rightarrow 0$, so that since $\mathcal{G}(\delta, \beta)$ is finite, $\mathcal{S}_{0} \rightarrow 0$. We conclude that for fixed $\delta$, there is no loss in $\frac{E_{b}}{N_{0} \text { min }}$ and $\mathcal{S}_{0}$ for high and low values of $\beta$.

We now consider the behavior of $\mathcal{D}(\delta, \beta)$ and $\mathcal{G}(\delta, \beta)$ for fixed $\beta$. In this case, for $\delta \ll 1$ we have that

$$
\delta \mathcal{F}(1 / \delta, \beta) \rightarrow \begin{cases}4 \beta, & \beta \leq 1 \\ 4, & \beta \geq 1\end{cases}
$$

so that

$$
\mathcal{D}(\delta, \beta) \rightarrow \begin{cases}\frac{1}{1-\beta}, & \beta \leq 1 \\ \frac{\beta}{\beta-1}, & \beta \geq 1\end{cases}
$$

and

$$
\mathcal{G}(\delta, \beta) \rightarrow \begin{cases}\frac{1}{3}(1+2 \beta), & \beta \leq 1 \\ 1, & \beta \geq 1 .\end{cases}
$$

For fixed $\beta$ and $\delta \gg 1, \mathcal{D}(\delta, \beta) \rightarrow 1$ and

$$
\mathcal{G}(\delta, \beta) \rightarrow \frac{1+2 \beta}{3+2 \beta} \text {. }
$$

Here we used the fact that from (75)

$\delta^{2} \mathcal{F}(1 / \delta, \beta)=4 \beta E\left\{\frac{\delta \lambda}{\lambda+\delta}\right\} \rightarrow 4 \beta E\{\lambda\}=4 \beta, \quad \delta \gg 1$. 


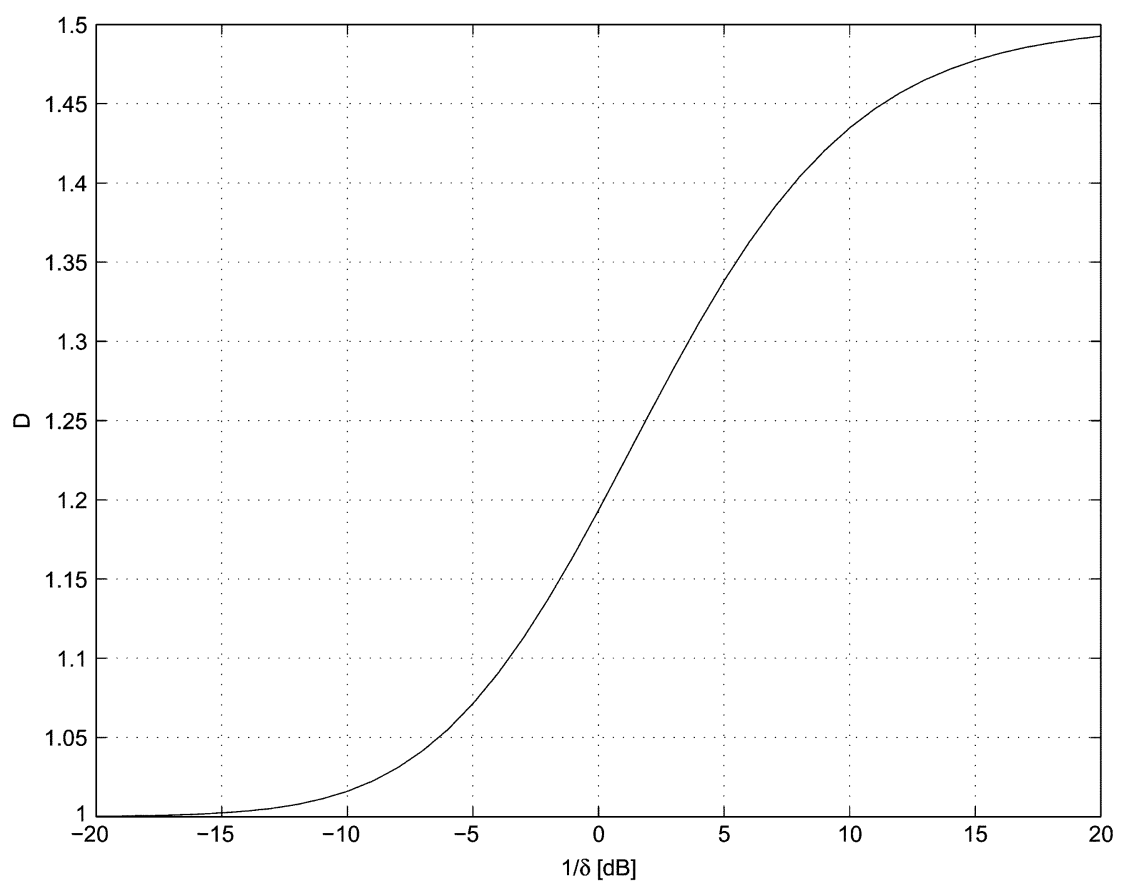

Fig. 14. $\mathcal{D}(\delta, \beta)$ of (117) as a function of $1 / \delta[\mathrm{dB}]$, for $\beta=3$.

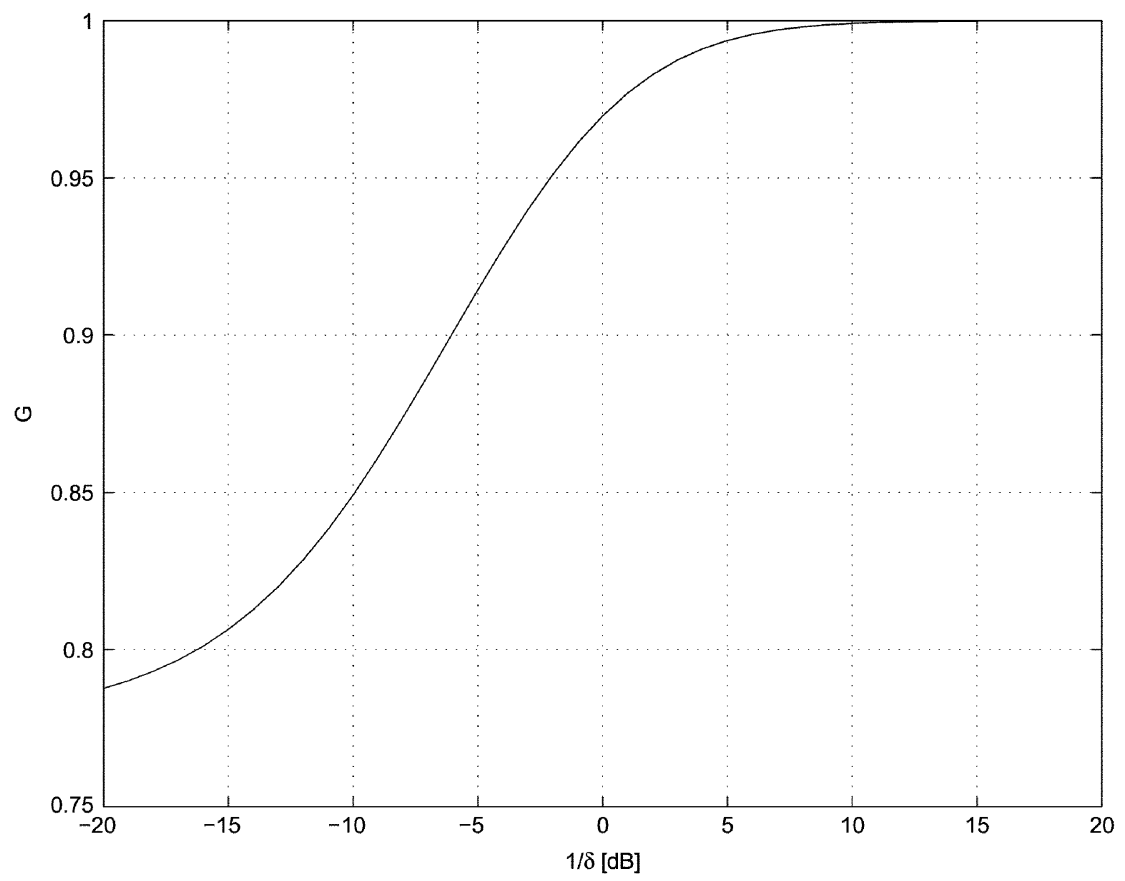

Fig. 15. $\mathcal{G}(\delta, \beta)$ of (119) as a function of $1 / \delta[\mathrm{dB}]$, for $\beta=3$.

The functions $\mathcal{D}(\delta, \beta)$ and $\mathcal{G}(\delta, \beta)$ as a function of $1 / \delta$ are illustrated in Figs. 14 and 15 respectively, for $\beta=3$.

\section{E. Robust MMSE-Based Receiver}

Using Theorem 5 we now propose a robust CSMU receiver in the case in which the SNR is not known exactly, whose performance is very close to that of the MMSE receiver that knows the SNR.

Suppose that the SNR, or equivalently, $\zeta$, is not known exactly, but is known to lie in an interval $L \leq \zeta \leq U$. Since the MMSE receiver depends explicitly on $\zeta$, it cannot be implemented if $\zeta$ is not known. One approach in this case is to implement the MMSE-based receiver of Theorem 5 with a specific choice of $\delta$. The simplest method is to choose $\delta=(U+L) / 2$ as the average of $\zeta$ in the uncertainty regime. However, as we now show, we can improve the performance and approach the performance of the MMSE receiver by choosing $\delta$ in a robust way.

Specifically, given a value of $\delta$, we may compute the loss in SINR performance, or the SINR regret, with respect to the 


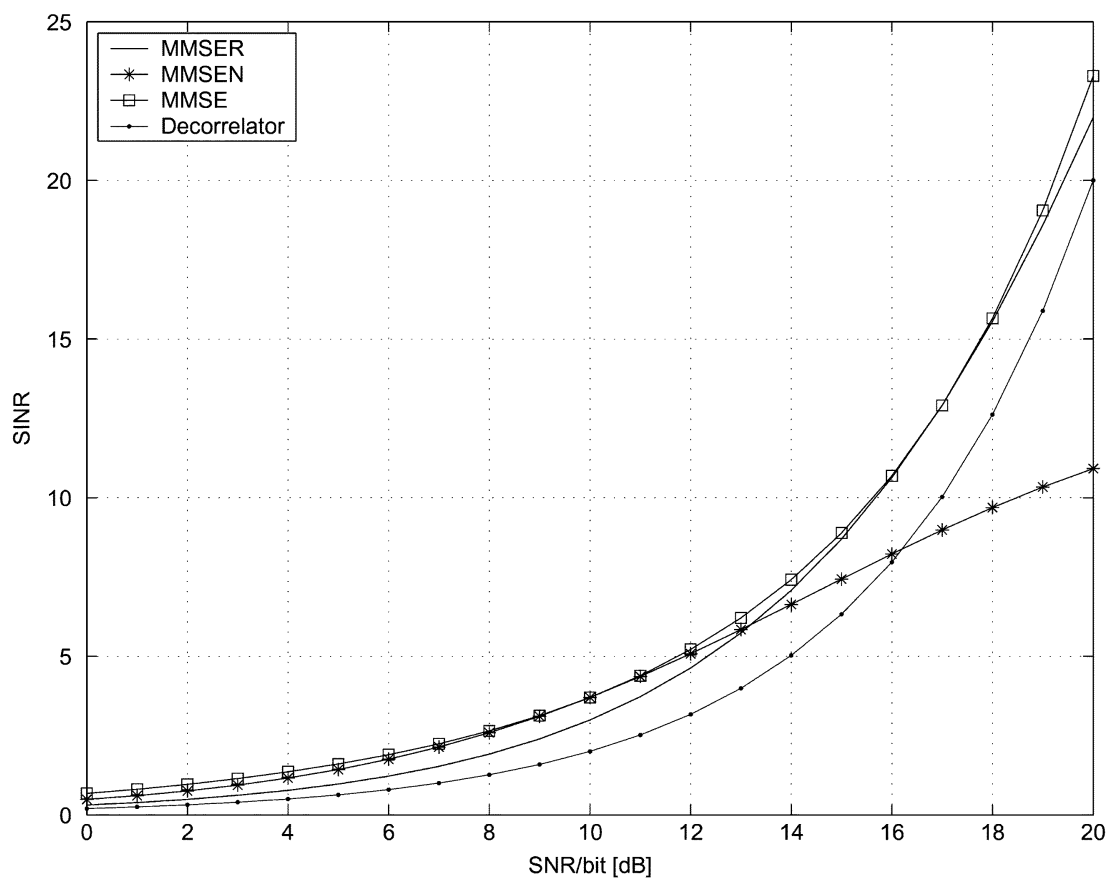

Fig. 16. SINR as a function of SNR, in the large-system limit, with equal-power users, random signatures, and $\beta=0.8$.

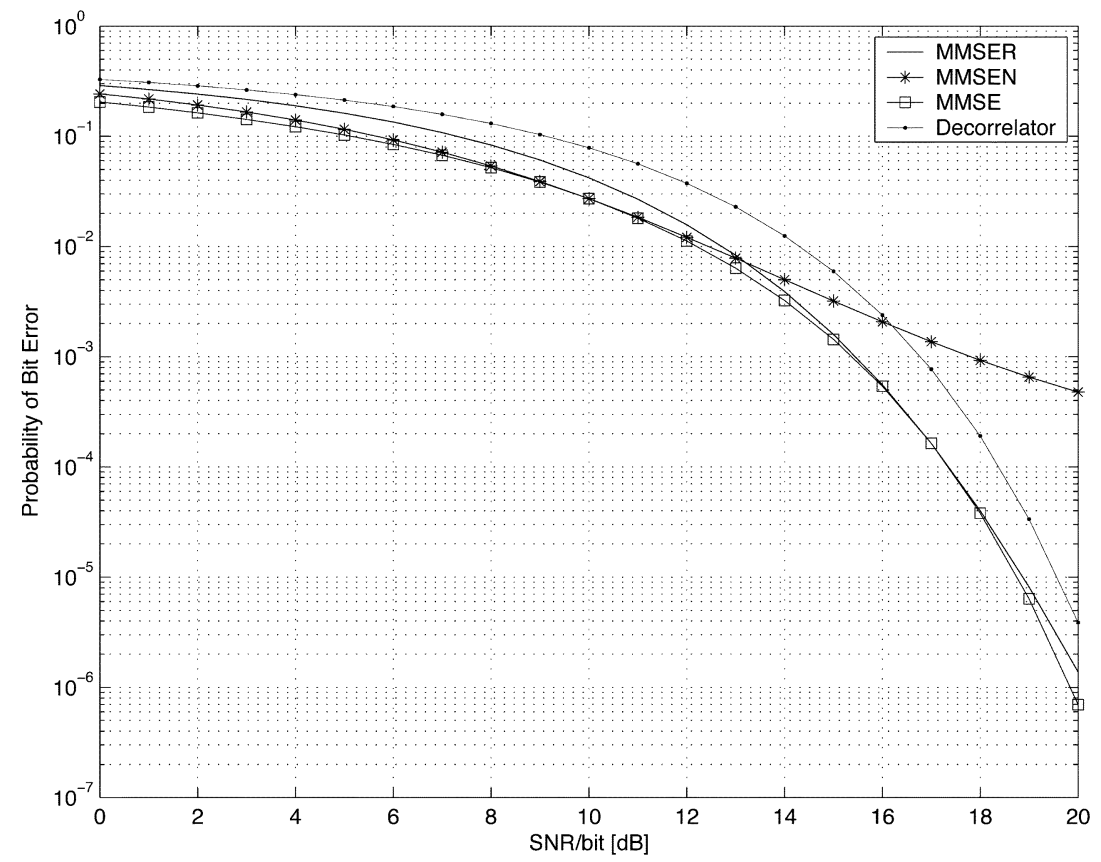

Fig. 17. Probability of bit error as a function of SNR, in the large-system limit, with equal-power users, random signatures, and $\beta=0.8$.

MMSE receiver for each value of $\zeta$. From Theorem 5, the regret is given by (128) at the bottom of the page. Clearly, $\mathcal{R}(\zeta, \zeta)=$ 0 , so that when $\zeta=\delta$ the regret is 0 . For all other values of $\zeta, \mathcal{R}(\zeta, \delta) \geq 0$. A receiver for which the regret is small across the uncertainty range in $\zeta$ will perform similarly to the MMSE receiver. To design such a receiver, we propose choosing $\delta$ such that the regret at both of the endpoints of the uncertainty region are equal. Since, in addition, the regret is equal to 0 at some point in the uncertainty region (the point for which $\zeta=\delta$ ), this will ensure that at any point in the uncertainty range the regret is not too large. Thus, we suggest choosing $\delta$ such that

$$
\mathcal{R}(U, \delta)=\mathcal{R}(L, \delta)
$$

$$
\mathcal{R}(\delta, \zeta)=\frac{1}{\zeta}-\frac{1}{4} \mathcal{F}(1 / \zeta, \beta)-\frac{1+\beta+\delta-\frac{1}{2} \delta \mathcal{F}(1 / \delta, \beta)}{\left(\frac{1}{\delta}-\frac{1}{4} \mathcal{F}(1 / \delta, \beta)\right)^{-1}\left(1+\beta+\zeta-\frac{1}{2} \delta \mathcal{F}(1 / \delta, \beta)\right)+\zeta-\delta}
$$


We refer to the resulting receiver as the Robust MMSE-based receiver (MMSER). Using (129), the value of $\delta$ can be computed numerically.

In Figs. 16 and 17, we plot the asymptotic SINR and the asymptotic BER, respectively, for $\beta=0.8$ using the MMSER receiver with $L=-15[\mathrm{~dB}]$ and $U=-5[\mathrm{~dB}]$. For comparison, we also plot the SINR and the BER using the MMSE-based receiver corresponding to $\delta=(U+L) / 2$, which we denote by MMSEN, and the MMSE receiver that knows $\zeta$. As can be seen in the figures, the performance of the robust MMSE receiver is very close to that of the MMSE receiver that knows the SNR, over the uncertainty interval. In contrast, choosing $\delta$ as the average value can deteriorate the performance, particulary at high SNR values.

We note, that a similar robust approach can be taken with respect to the MFD receiver of Section V-C in the case in which the SNR is not known completely.

\section{CONCLUSION}

In this paper, we developed a new class of linear multiuser receivers for CDMA channels, referred to as the CSMU receiver, which is based on a CSLS estimate of the users' coded symbols. The MMSE, decorrelator, MF, OMU, and Müller-Verdú receivers are all shown to be special cases of the CSMU receiver. Based on the properties of the CSLS estimator, we developed several different interpretations of the CSMU receiver, which provide further insight into its properties. We then analyzed the large system performance of this receiver, for a broad class of output covariance matrices $\boldsymbol{R}$. Specifically, we developed closed-form expressions for the output SINR, the minimal energy-per-information bit required to transmit information reliably, and the wideband slope of the CSMU receiver.

We then considered a special case of the CSMU receiver, in which the channel SNR is not known precisely, and the receiver is matched to an output covariance which has a structure similar to that of the output covariance of the MMSE receiver. The resulting receiver is shown to be a mismatched MMSE receiver, in which the actual channel SNR is replaced by an estimated SNR. Analysis of this receiver demonstrates that without knowledge of the channel SNR, the performance of the mismatched MMSE receiver is often very close to that of the MMSE receiver. We then suggested a robust receiver whose performance is close to that of the MMSE receiver over an uncertainty range in the SNR.

In this paper we analyzed the performance of the CSMU receiver in the case of accurate power control, and for covariance matrices $\boldsymbol{R}$ that commute with $\boldsymbol{S}^{*} \boldsymbol{S}$. An interesting direction for future research is to generalize the performance analysis to unequal-power users, and a broader class of covariance matrices $\boldsymbol{R}$. It would also be of interest to investigate the performance analysis with binary symbols.

\section{REFERENCES}

[1] S. Verdú, Multiuser Detection. New York: Cambridge Univ. Press, 1998.

[2] - "Minimum probability of error for asynchronous Gaussian multiple-access channels," IEEE Trans. Inf. Theory, vol. IT-32, no. 1, pp. 85-96, Jan. 1986.
[3] — - "Optimum multi-user asymptotic efficiency," IEEE Trans. Commun., vol. 38, no. 4, pp. 496-508, Apr. 1990.

[4] D. N. C. Tse and S. V. Hanly, "Linear multiuser receivers: Effective interference, effective bandwidth, and user capacity," IEEE Trans. Inf. Theory, vol. 45, no. 2, pp. 641-657, Mar. 1999.

[5] D. N. C. Tse and O. Zeitouni, "Linear multiuser receivers in random environments," IEEE Trans. Inf. Theory, vol. 46, no. 1, pp. 171-188, Jan. 2000

[6] Z. Xie, R. Short, and C. Rushforth, "A family of suboptimum detectors for coherent multiuser communications," IEEE J. Sel. Areas Commun., vol. 8, no. 4, pp. 683-690, May 1990.

[7] U. Madhow and M. Honig, "MMSE interference suppression for directsequence spread-spectrum CDMA," IEEE Trans. Commun., vol. 42, no. 12, pp. 3178-3188, Dec. 1994.

[8] P. Rapajic and B. Vucetic, "Adaptive receiver structures for asynchronous CDMA systems," IEEE J. Sel. Areas Commun., vol. 12, no. 4, pp. 685-697, May 1994.

[9] M. Rupf, F. Tarköy, and J. L. Massey, "User-separating demodulation for code-division multiple-access systems," IEEE J. Sel. Areas Commun., vol. 12, no. 5, pp. 786-795, Jun. 1994.

[10] R. Lupas and S. Verdú, "Linear multiuser detectors for synchronous code-division multiple-access channels," IEEE Trans. Inf. Theory, vol. 35, no. 1, pp. 123-136, Jan. 1989.

[11] - "Near-far resistance of multiuser detectors in asynchronous channels," IEEE Trans. Commun., vol. 38, no. 4, pp. 496-508, Apr. 1990

[12] Y. C. Eldar and A. M. Chan, "An optimal whitening approach to linear multiuser detection," IEEE Trans. Inf. Theory, vol. 49, no. 9, pp. 2156-2171, Sep. 2003.

[13] Y. C. Eldar and A. V. Oppenheim, "Orthogonal multiuser detection," Signal Process., vol. 82, pp. 321-325, Feb. 2002.

[14] D. Guo, L. K. Rasmussen, S. Sun, and T. J. Lim, "A matrix-algebraic approach to linear parallel interference cancellation in CDMA," IEEE Trans. Commun., vol. 48, no. 1, pp. 152-161, Jan. 2000.

[15] R. R. Müller and S. Verdú, "Design and analysis of low-complexity interference mitigation on vector channels," IEEE J. Sel. Areas Commun., vol. 19, no. 8, pp. 1429-1441, Aug. 2001.

[16] Y. C. Eldar and A. V. Oppenheim, "Covariance shaping least-squares estimation," IEEE Trans. Signal Process., vol. 51, no. 3, pp. 686-697, Mar. 2003.

[17] Y.C. Eldar, "Quantum signal processing," Ph.D. dissertation, MIT, Cambridge, MA, Dec. 2001.

[18] S. Verdú and S. Shamai (Shitz), "Multiuser detection with random spreading and error-correction codes: Fundamental limits," in Proc. 35th Allerton Conf. Communication, Control, and Computing, Monticello, IL, Sep./Oct. 1997, pp. 470-482.

[19] - "Spectral efficiency of CDMA with random spreading," IEEE Trans. Inf. Theory, vol. 45, no. 3, pp. 622-640, Mar. 1999.

[20] Y. C. Eldar and A. M. Chan, "On the asymptotic performance of the decorrelator," IEEE Trans. Inf. Theory, vol. 49, no. 9, pp. 2309-2313, Sep. 2003.

[21] S. Shamai (Shitz) and S. Verdú, "The impact of frequency-flat fading on the spectral efficiency of CDMA," IEEE Trans. Inf. Theory, vol. 47, no. 5, pp. 1302-1327, May 2001.

[22] S. Verdú, "Spectral efficiency in the wideband regime," IEEE Trans. Inf. Theory, vol. 48, no. 6, pp. 1319-1343, Jun. 2002.

[23] G. H. Golub and C. F. Van Loan, Matrix Computations, 3rd ed. Baltimore, MD: Johns Hopkins Univ. Press, 1996.

[24] Y. C. Eldar, "On geometric properties of the decorrelator," IEEE Commun. Lett., vol. 6, no. 1, pp. 16-18, Jan. 2002.

[25] Y. C. Eldar and A. V. Oppenheim, "Quantum signal processing," IEEE Signal Processing Mag., vol. 19, no. 6, pp. 12-32, Nov. 2002.

[26] - "MMSE whitening and subspace whitening," IEEE Trans. Inf. Theory, vol. 49, no. 7, pp. 1846-1851, July 2003.

[27] T. L. Marzetta and B. M. Hochwald, "Capacity of a mobile multipleantenna communication link in Rayleigh flat fading," IEEE Trans. Inf. Theory, vol. 45, no. 1, pp. 139-157, Jan. 1999.

[28] R. Muirhead, Aspects of Multivariate Statistical Theory. New York: Wiley, 1982.

[29] Z. D. Bai and Y. Q. Yin, "Limit of the smallest eigenvalue of a large dimensional sample covariance matrix," Ann. Probab., vol. 21, pp. 1275-1294, 1993.

[30] I. S. Gradshteyn and I. M. Ryzhik, Table of Integrals, Series, and Products. New York: Academic, 1980.

[31] D. Guo, S. Verdú, and L. K. Rasmussen, "Asymptotic normality of linear multiuser receiver outputs," IEEE Trans. Inf. Theory, vol. 48, no. 12, pp. 3080-3095, Dec. 2002. 